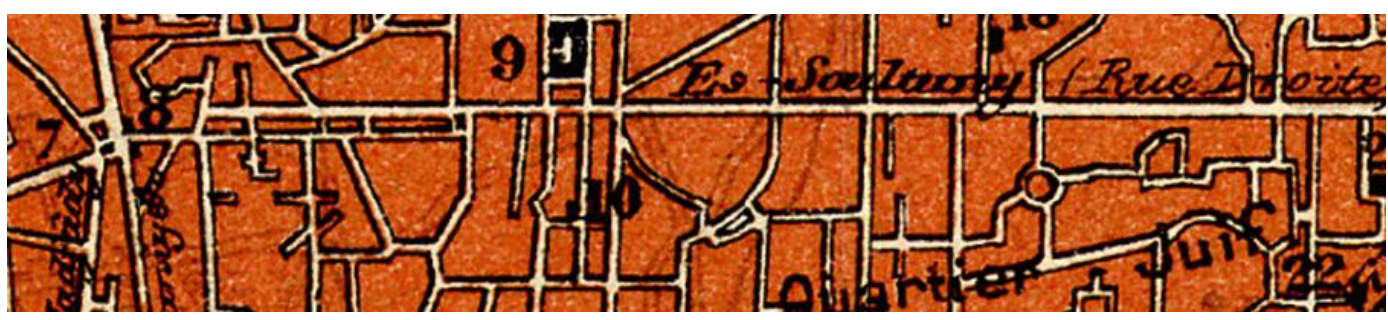

\title{
Corpus cartographique pour l'histoire de Damas, Syrie, à la fin de la période ottomane (1760-1924)
}

\author{
D'après : Jean-Luc Arnaud, "Corpus cartographique pour l'histoire \\ de Damas, Syrie, à la fin de la période ottomane (1760-1924)", \\ Imago Mundi, The International Journal for the History of \\ Cartography, 53, 2001, p. 46-70.
}

\section{Résumé}

De manière générale, la cartographie des villes de l'empire ottoman est peu abondante. Au dix-neuvième siècle, malgré plusieurs réformes administratives, l'autorité publique ne recourait pas à des documents cartographiques pour organiser les travaux d'édilité tandis que les étrangers rencontraient les plus grandes difficultés pour établir des représentations de ces villes. Damas constitue une exception à ce tableau. On dispose de plusieurs cartes antérieures à l'établissement du mandat français (1920). Les auteurs étrangers sont les plus nombreux et les plus fiables ; le plus souvent archéologues ou historiens, ils ont dressé des documents partiels pas toujours faciles à évaluer. Cependant, et malgré la faiblesse de son volume (sept documents), le corpus des premières cartes de Damas constitue une source essentielle pour l'histoire du développement de la ville entre le milieu du dix-neuvième siècle et l'établissement du pouvoir mandataire.

\section{Abstract}

Maps of towns in the Ottoman Empire are rare. In the nineteenth century, despite a number of administrative reforms, the public authorities did not turn to cartography in the development of the built-up area, and foreigners encountered major difficulties when they attempted to represent the town cartographically. The exception was Damascus. Several maps of the city pre-date the establishment of the French Mandate (1920), although they are of unequal quality. Foreign authors, usually archaeologists or historians, accounted for both the majority and the most reliable of the maps, although they did not show the whole city and the maps are not always easy to interpret. However, despite the small number (seven) of these maps, the corpus constitutes an essential source for the history of the city's 
spatial development between the middle of the nineteenth century and the setting up of the Mandate

Kurzfassung

Karten von Städten des Osmanischen Reiches sind im Allgemeinen rar. Im 19 Jahrhundert, wurden ungeachtet einiger administrativer Reformen bei der Erschließung besiedelter Gebiete kaum kartographische Hilfsmittel verwendet. Versuche von Ausländem diese Städte kartographisch darzustellen, stießen ihrerseits auf erhebliche Probleme. Damaskus bildet hiervon die Ausnahme: Man kennt eine größere Zahl von qualitativ unterchiedlichen Karten aus der Zeit vor dem französischen Mandat (1920). Ausländer bearbeiteten nicht nur den Großteil dieser Karten, sondem auch die zuverlässigsten von ihnen. Die meisten Autoren waren Archäologen oder Historiker und sie zeichneten meist nur Detailpläne, die nicht immer einfach zu interpretieren sind. Obwohl es sich mit sieben Karten nur um einen kleinen Bestand handelt, stellen diese frühen Karten von Damaskus eine wichtige. Quelle für die Geschichte der Stadtentwicklung zwischen der Mitte des 19. Jahrhunderts und der Einrichtung der Mandats-Regierung dar. 


\section{Corpus cartographique pour l'histoire de Damas, Syrie, à la fin de la période ottomane (1760-1924)}

De manière générale, les sources cartographiques pour les villes de l'Empire ottoman sont assez rares et de qualité assez médiocre jusqu'au début du vingtième siècle. Il existe cependant plusieurs exceptions à cette règle. En Egypte tout d'abord, ou les plus grandes villes ont été relevées avec précision dès la fin du dix-huitième siècle à la faveur de l'expédition française ${ }^{1}$; Damas en constitue une seconde, moins connue ${ }^{2}$. Ce chef-lieu de province jusqu'à l'issue de la Première Guerre Mondiale n'a pas été l'objet d'une cartographie abondante durant la période ottomane. Cependant, un plan datant de la seconde moitié du dix-neuvième siècle, attribué à Emmanuel Guillaume Rey, présente un intérêt paradoxal (voir fig. 8) ) $^{3}$ Il semble de trop bonne qualité pour la date à laquelle il aurait été dressé (1860), non pour des raisons techniques, car ce plan ne résulte pas d'une prouesse en regard des méthodes disponibles en Europe, mais plus simplement par le fait que son auteur a disposé des moyens de visiter l'ensemble de la ville et de ses faubourgs et de leur porter la même attention. Rey n'était ni mandaté ni protégé par les autorités publiques ; on l'examinera, au contraire de leurs confrères européens, les édiles ottomans portaient peu d'intérêt aux questions de cartographie urbaine à ce moment-là.

\section{Des conditions difficiles}

Habituellement, les voyageurs européens rencontrent dans les villes de l'Empire ottoman les plus grandes difficultés, pour tout ce qui concerne les travaux iconographiques ou cartographiques, face aux réticences de la population et des autorités. Les exemples sont multiples. A la fin du dix-huitième siècle, Cornelius Le Brun prétend avoir risqué sa vie pour dresser un panorama de Jérusalem ${ }^{4}$. Au cours du siècle suivant, Carsten Niebuhr renonce à utiliser son astrolabe pour effectuer un relevé d'Alexandrie ${ }^{5}$ tandis que les savants de l'expédition d'Egypte ne peuvent pas déployer leurs instruments sans être protégés par une escorte militaire ${ }^{6}$. Cette situation semble se détendre au cours du dix-neuvième siècle. Pour lstanbul, Antoine Ignace Melling, qui dresse in-situ des dessins détaillés de la ville

\footnotetext{
1. Anne Godlewska, The Napoleonic Survey of Egypt. A Mastemiece of Cartographie Compilation and Early Nineteenth-Century Fieldwork, Cartographica 25, Monograph 38-39, Toronto, University of Toronto Press, 1988.

2. De manière générale, la cartographie des villes de cette région reste à étudier, les références sont peu abondantes. Pour Beyrouth, on consultera Cartographie de Beyrouth, Beyrouth, Centre d'études et de recherches sur le Moyen-Orient contemporain, Al-Layali, 1995. Pour Alep, on ne dispose pas d'inventaire mais on se réfèrera à Jean-Claude David, "Le plan d'Alep du consul Rousseau (début du XIXe), espace représenté, espace connu, espace pratiqué", dans Etudes sur les villes du Proche-Orient XVIe-XIXe siècle. Hommages à André Raymond, Damas, Institut français d'études arabes de Damas, 2001, p. 151-160.

3 . "Plan de Damas et de ses faubourgs", dans Adrien Chauvet et Emile Isambert, Itinéraire descriptif historique et archéologique de l'Orient par le Dr E. Isambert, tome troisième Syrie, Palestine..., Paris, Librairie Hachette, s.d. (éd. de 1882 (. . .) mis au courant en 1890), encarté face à la page 636.

${ }^{4}$. Cornelius Le Brun, Voyage au Levant c'est-à-dire dans les principaux endroits de l'Asie mineure dans les isles de Chio, de Rhodes, de Chypre, etc., Paris, Guillaume Cavelier, 1714, p. 286-287.

5 . Carsten Niebuhr, Voyage en Arabie et en d'autres pays circonvoisins par C. Niebuhr, traduit de l'allemand, Amsterdam, chez S. J. Baalde, Utrecht, chez J. Van Schoonhoven et comp., 1776, vol. 1, p. 39.

6 . Pierre Jacotin, "Mémoire sur la construction de la carte de I'Egypte", dans Description de I'Egypte, Paris, Imprimerie impériale, 1822, état moderne, tome 2, 2e partie, p. 11.
} 
vers 1810 , en constitue le témoin. Il explique que vingt ans plus tôt, on ne lui aurait pas laissé effectuer les mêmes travaux ${ }^{7}$.

Nous ne disposons pas d'informations semblables pour les villes syriennes. A Alep, par exemple, le consul Rousseau, qui relève un plan de la ville au cours des années 1810, figure avec plus de précision les quartiers habités par les chrétiens que ceux occupés par les musulmans ${ }^{8}$. Pour sa part, Damas jouit d'une solide réputation d'intolérance, les voyageurs ne manquent jamais de rappeler son fanatisme et la nécessité d'être vêtu du costume local pour circuler en ville ${ }^{9}$. Ces témoignages deviennent moins fréquents au cours de la première moitié du dix-neuvième siècle ; Emile Gentil qui visite Damas au début des années 1850 en rend compte. Il explique qu'il est devenu possible depuis quelques années de parcourir la ville en habit européen mais il relève aussi les multiples insultes qui lui sont adressées à l'occasion de ses visites ${ }^{10}$.

Or, le plan de Rey représente de manière précise les impasses les plus reculées. S'il est indiscutable qu'il résulte d'un relevé, c'est sa datation qui est en cause. En effet, ce plan a été publié au début des années 1880 mais aucune indication ne précise avec certitude la date de son élaboration. Par sa qualité, il constitue une source majeure pour l'histoire de l'urbanisation de Damas ; c'est le premier document qui permet de tracer son périmètre complet de manière détaillée. Il s'avère ainsi nécessaire de le dater avec précision. En outre, malgré ses qualités, ce plan présente des défauts qui imposent une critique minutieuse en le replaçant dans le contexte de sa production et en le confrontant à ceux qui le précèdent et qui le suivent. Aussi, cet article traite-t-il de l'ensemble des plans de Damas depuis la seconde moitié du dix-huitième siècle jusqu'au début de la période mandataire (1920). Alors que la plus grande part des plans antérieurs à cette date a été dressée par des étrangers indépendants du pouvoir, à partir de l'établissement du Mandat français, les documents cartographiques sont produits par l'autorité publique ; leur qualité s'améliore de manière substantielle, ils sont triangulés suivant les règles de l'art tandis que leur volume s'accroît. Il s'agit alors d'une autre histoire qui offre une place très différente aux questions d'épistémologie.

\section{Damas: une ville en mutations}

Au cours de la période couverte par ces documents - de la fin du dix-huitième au début du vingtième siècle -, l'agglomération de Damas connaît d'importantes extensions et une recomposition de son organisation spatiale (fig. 1). En 1860, la zone urbanisée regroupe 420 hectares et la ville n'a pas connu de développement remarquable depuis la fin du dixhuitième siècle; au début de la période mandataire, cette surface atteint 570 hectares. Alors que le faubourg du Mîdân, organisé en direction du sud autour de la route de Jérusalem et de La Mecque, a longtemps été considéré comme le principal vecteur de croissance de l'agglomération ${ }^{11}$, les extensions de la fin du dix-neuvième siècle sont orientées dans d'autres directions. C'est en particulier vers l'ouest, c'est-à-dire vers Beyrouth, à la faveur de l'ouverture d'une route carrossable entre Damas et cette ville en 1863, que l'agglo-

\footnotetext{
7 . Antoine Ignace Melling et Jean Denis Barbié du Bocage, Voyage pittoresque de Constantinople et des rives du Bosphore d'après les dessins de M. Melling, Paris, Treuttel et Würtz, 1819, notice de la pl. 22.

8 . J.-C. David, op. cit.

9 . Constantin François Chasseboeuf, comte de Volney, Voyage en Egypte et en Syrie pendant les années 17831784 et 1785, Paris, Bossange frères, 1822, 5e éd., p. 146.

10 . Emile Gentil, Souvenirs d'orient, Metz, Paris, Jules Verronnais, 1855, p. 113-114 et 126.

11 . André Raymond, Grandes villes arabes à l'époque ottomane, Paris, Sindbad, 1985, p. 48.
} 


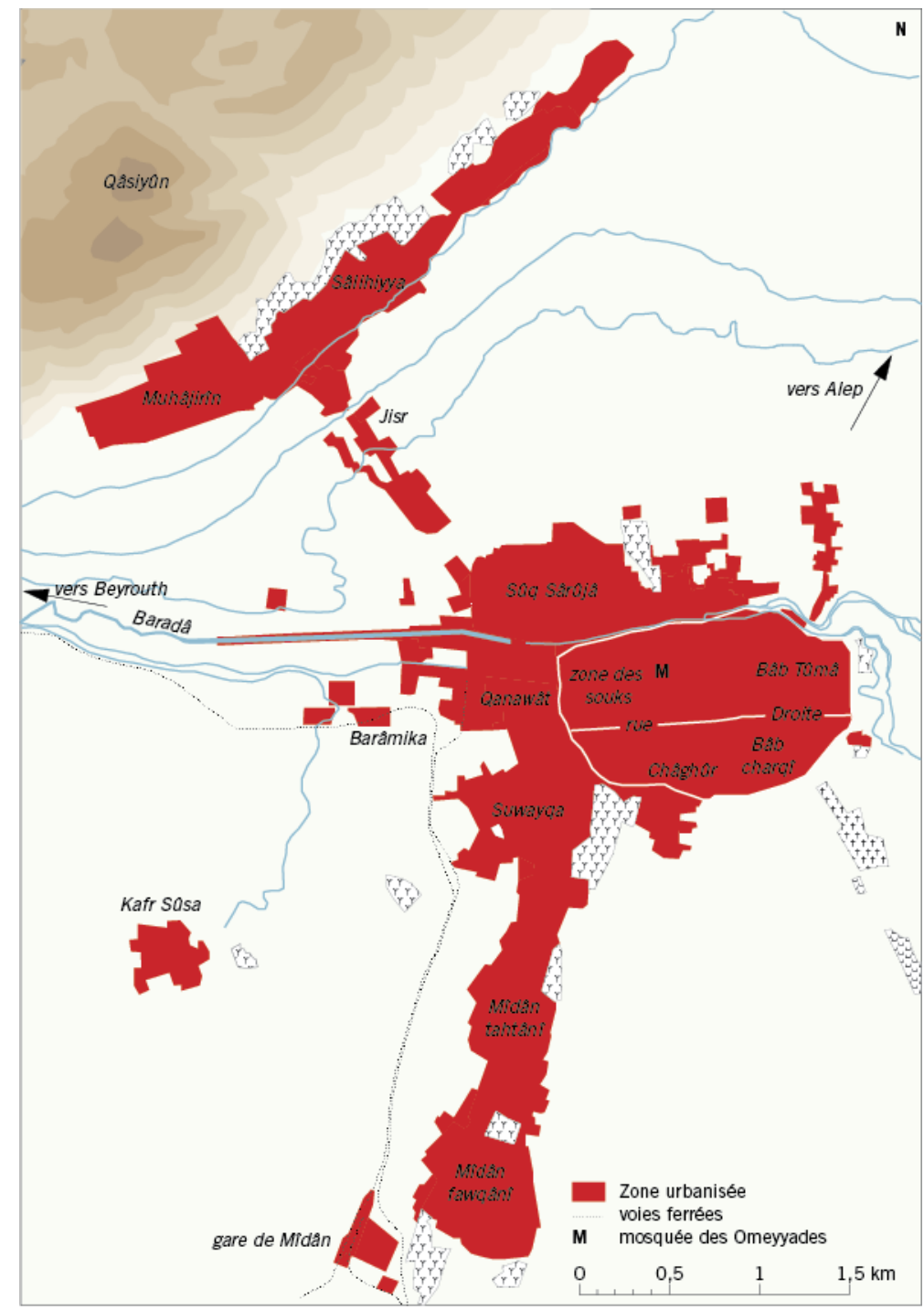

Fig. 1. Damas en 1918. Situation des lieux cités et emprise de l'urbanisation à la veille du Mandat français. Document établi par l'auteur d'après Damas, 1:20 000 (voir note 32) pour la topographie ; L. Vibert, Plan de Damas (voir note 32), pour le tracé des voies ferrées et du tramway ; Al-cham dimachq... (voir note 31) pour les limites de l'urbanisation.

mération s'étale le plus largement ${ }^{12}$. Les établissements de l'autorité publique, mis en place ou renouvelés dans le cadre des réformes administratives de l'Empire ottoman (tanzimat) ${ }^{13}$ sont aussi installés dans les nouveaux quartiers de l'ouest qu'ils contribuent à promouvoir. A la veille du Mandat français, on y trouve le palais du gouverneur, le siège de la municipalité, celui de la police, plusieurs écoles, des casernes, etc. Les terminus des nouvelles voies ferrées qui mettent Damas en relation plus étroite avec la plaine céréalière du Hauran (vers le sud), La Mecque et Beyrouth (son port) sont aussi localisés dans ces quartiers. Dans une moindre mesure, vers le nord, la route d'Alep constitue aussi un

12. A propos de l'ouverture de cette route et de ses enjeux, voir Leila Fawaz, "The Beirut-Damascus road: connecting the Syrian coast to the interior in the 19th century", dans Thomas Philipp et Birgit Schaebler (éd.), The Syrian Land: Process of Integration and Fragmentation in Bilad al-Sham from the 18th to the 20th Century, Stuttgart, Franz Steiner, 1998, p. 19-28.

13 . L'édit de Gülhane de 1839 puis le Khan i hümayûn publié en 1856, constituent la base de ces réformes. Paul Dumont, "La période des Tanzîmât (1839-1878)", dans Robert Mantran (dir.), Histoire de l'Empire ottoman, Paris, Fayard, 1989, p. 492-508. 
nouveau vecteur de croissance urbaine. Il prolonge hors les murs le quartier de prédilection des chrétiens: Bâb Tûmâ on y trouve plusieurs établissements de cette communauté. Enfin, durant cette période, le volume de la population connaît une augmentation substantielle ${ }^{14}$. Dans ce contexte, les plans successifs constituent des sources précieuses pour l'histoire de l'urbanisation. Ce ne sont pas les seuls documents disponibles pour en rendre compte mais, au contraire des archives écrites, les sources cartographiques permettent d'effectuer une pesée globale des mutations et d'en analyser la répartition géographique à l'échelle de l'ensemble de l'agglomération ${ }^{15}$.

Pour organiser le corpus, les huit documents étudiés ont été réparti en deux générations en fonction de leur date d'élaboration. La coupure entre les deux correspond à la fin des années 1850, les plans antérieurs à cette date sont sommaires et incomplets. Ceux de Carsten Niebuhr (1760), de Alfred von Kremer (1854) et de Josias Leslie Porter (1855) constituent cette première génération. Les suivants, dont le plan de Rey est le plus ancien, résultent de travaux plus accomplis mais ils présentent des défauts qu'il n'est pas inutile d'évaluer.

\section{Première génération (1760-1855)}

\section{Carsten Niebuhr (1760)}

Le plus ancien véritable plan de Damas, c'est-à-dire une projection parallèle verticale, est certainement celui dressé par Carsten Niebuhr au cours des années 1760 (fig. $2)^{16}$. L'auteur, arpenteur de formation, accompagne alors une expédition scientifique danoise au sein de laquelle il est chargé de la cartographie ${ }^{17}$. Son livre ne compte pas moins de quatorze plans de villes, depuis Constantinople jusqu'à Damas, en passant par Le Caire, Sanaa, Chiraz et Bagdad ${ }^{18}$. Niebuhr ne dit rien des conditions dans lesquelles il a effectué le relevé de Damas mais il y a séjourné peu de temps; il est probable qu'il a procédé, comme pour la plupart des autres villes, au pas et à la boussole ${ }^{19}$. Ce document est pour le moins sommaire, il figure la position des vestiges encore visibles de l'enceinte, le périmètre de la zone agglomérée et trois ou quatre rues importantes. Si les faubourgs nord et ouest n'ont pas été oubliés, vers le sud, celui de Mîdân semble pratiquement inexistant alors qu'il s'étend sur plus de deux kilomètres le long d'une voie importante ponctuée par de nombreux édifices très antérieurs à la seconde moitié du dix-huitième siècle (fig. 3$)^{20}$. Il faut attendre

14. Alors qu'elle regroupe environ 100000 habitants au milieu du dix-neuvième siècle, l'agglomération de Damas en compte 150000 à la veille de la Première Guerre Mondiale. Jean-Luc Arnaud, "La population de Damas à la fin de la période ottomane", Annales de démographie historique n 1-2001, p. 177-207.

15 . Les archives relatives aux mutations foncières sont particulièrement abondantes, elles regroupent plus d'un millier de volumes pour la période qui m'intéresse. Brigitte Marino et Tomoki Okawara, Catalogue des registres des tribunaux ottomans conservés au centre des archives de Damas, Damas, Institut français d'études arabes de Damas, 1999.

16 . "Plans de la ville d'Orfa et de Damas", dans Carsten Niebuhr, Voyage en Arabie et en d'autres pays circonvoisins par C. Niebuhr, traduit de l'allemand, Amsterdam, chez S. J, Baalde, Utrecht, chez Barthelemy Wild, 1780, vol. 2, pl. 51.

17. Sur cette expédition, voir Torkild Hansen, La mort en Arabie. Une expédition danoise 1761-1767, trad. du danois, Arles, Actes Sud, 1988.

18 . Pour une évaluation globale de la production cartographique de C. Niebuhr, voir Ian W. J. Hopkins, "The Maps of Carsten Niebuhr: 200 years after", Cartographic Journal n 4, 1967, p. 115-118.

19 . C. Niebuhr, op. cit., vol. 1, p. 19.

${ }^{20}$. Damas extra-muros, présentation et analyse des édifices répertoriés, Midan Sultani, Damas, Institut français d'études arabes de Damas, 1994. 


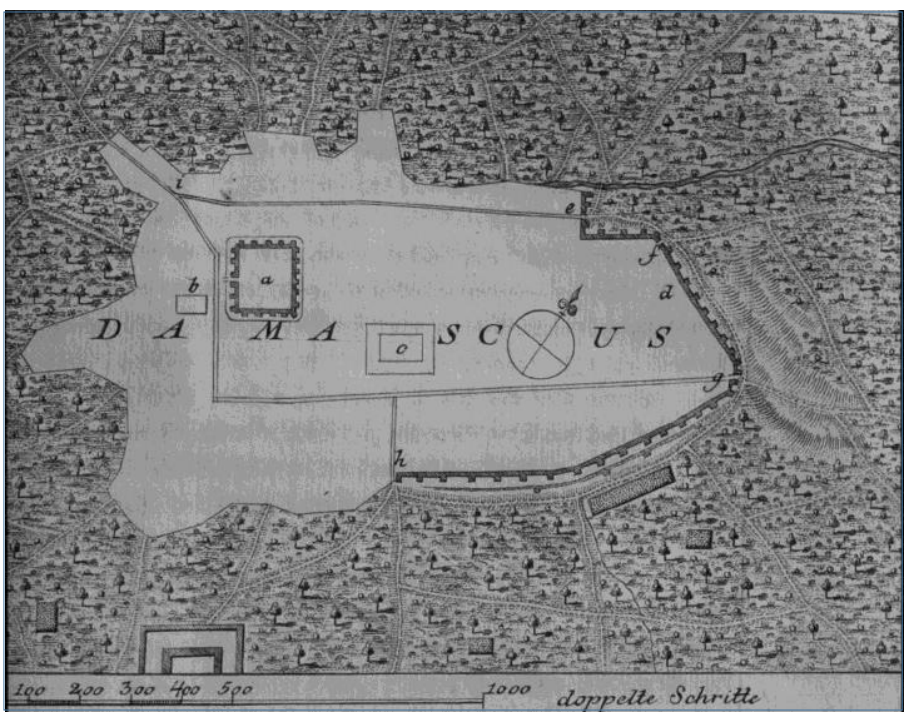

Fig. 2. Plan de Carsten Niebuhr, 1760. L'interruption de la zone urbanisée vers le sud avant le cadre de la carte témoigne du peu d'attention porté par Niebuhr au périmètre de l'agglomération. Extrait de "Plans de la ville d'Orfa et de Damas" (voir note 16), reproduit avec l'autorisation de l'Institut français d'études arabes de Damas.

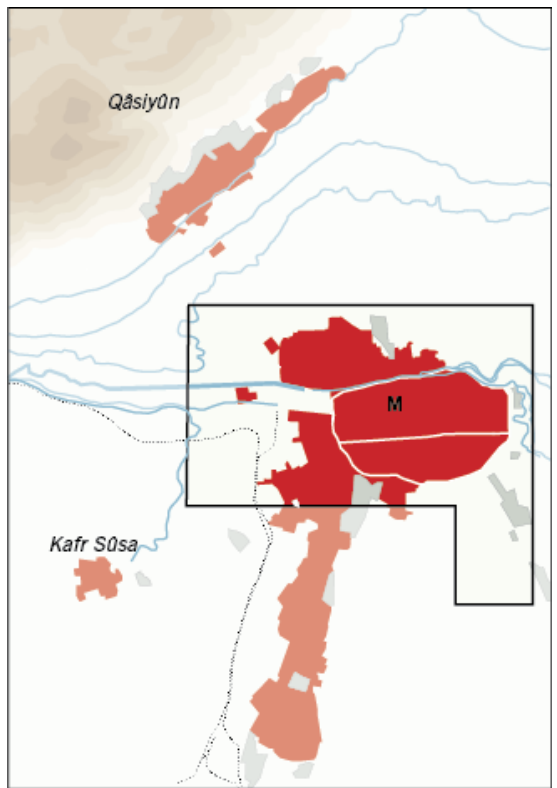

Fig. 3. Périmètre approximatif du plan de Carsten Niebuhr. Alors que, dans son texte, Niebuhr considère la surface urbanisée de chaque ville comme un indicateur majeur du volume de sa population, à Damas, il semble ignorer la plus grande part du faubourg sud. Document établi par l'auteur.

longtemps un document de meilleure qualité car c'est pratiquement un siècle plus tard que de nouveaux plans de Damas sont dressés.

Alors que la pénurie est patente pour la première moitié du dix-neuvième siècle, trois documents sont relevés en moins de dix ans autour des années 1850. Alfred von Kremer, un jeune étudiant Viennois qui séjourne deux ans à Damas au tournant des années1850 et en dresse un plan, publie le premier ${ }^{21}$. L'année suivante, Josias Leslie Porter, un religieux anglais qui vient de passer cinq ans au proche Orient, joint un plan de Damas à la relation

${ }^{21}$. Alfred von Kremer, Topographie von Dasmascus, Vienne, 1854 et 1855. Deux fascicules de 51 et 37 pages. Le second comporte un document cartographique sans titre suivi d'une liste de renvois correspondants aux numéros portés sur le document. L'auteur a séjourné à Damas au cours des années 1849-1850 et 1851. 


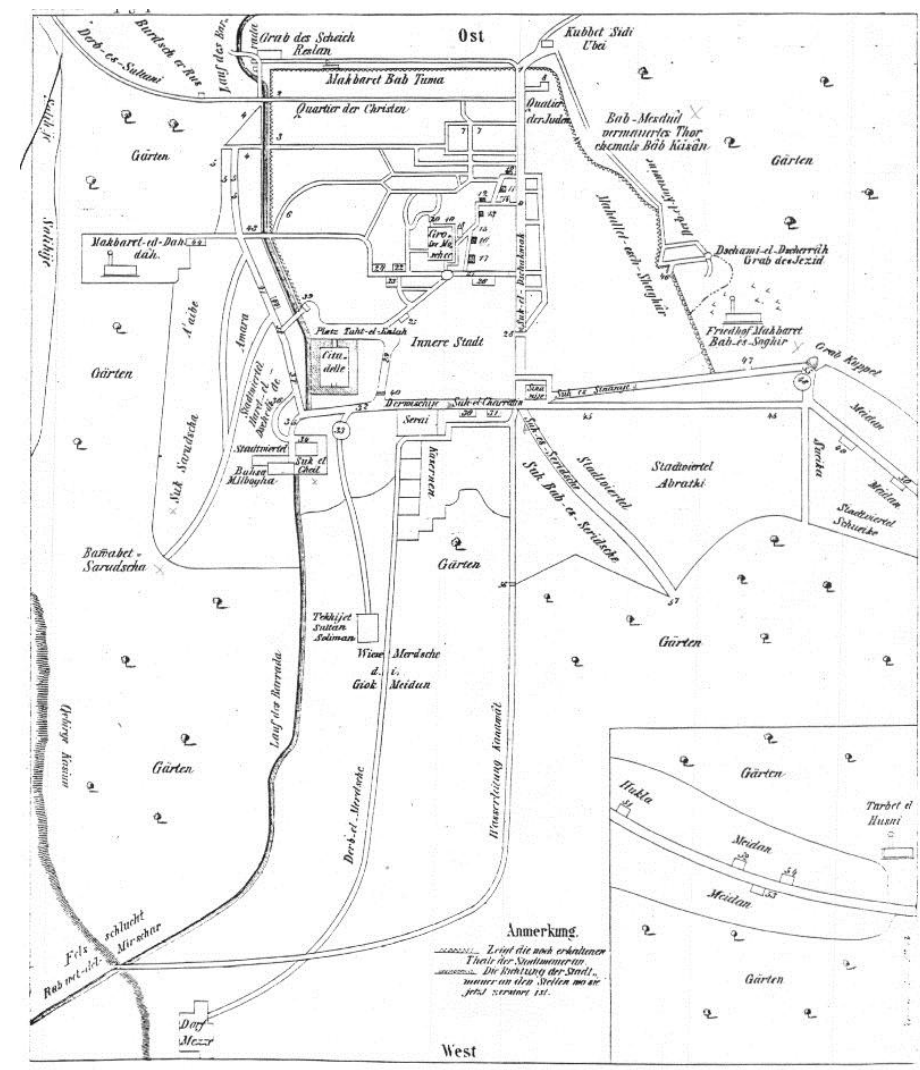

Fig. 4. Plan de Alfred von Kremer, 1854. Ce plan, composé par addition d'itinéraires, était tout d'abord destiné au repérage des édifices et des lieux présentant un intérêt archéologique. Document encarté dans Kremer, Topographie... (voir note 21), reproduit avec l'autorisation de l'Institut français d'études arabes de Damas.

de son voyage ${ }^{22}$ le troisième est le plan de Rey. Ces trois documents sont de qualité inégale, mais, malgré leur caractère parfois schématique, ils ne sont en aucun cas fantaisistes.

\section{Alfred von Kremer (1854)}

Alfred von Kremer est tout d'abord orientaliste, avant de devenir diplomate et homme politique, il effectue un séjour de deux années à Damas pour en explorer les bibliothèques. Compte tenu de sa formation et de ses centres d'intérêt, il ignore les techniques élémentaires de relevé cartographique et cette lacune se ressent fortement à la consultation de son plan (fig. 4). Kremer est cependant bon observateur quant à l'organisation de l'espace et, au contraire de Niebuhr, il a eu le loisir de visiter longuement la ville ${ }^{23}$.

Le document cartographique qu'il publie n'est pas un véritable plan. L'orientation y est indiquée sans rose ni flèche mais seulement par les désignations Est et Ouest placées en haut et en bas du document ; en outre, il ne comporte pas d'échelle et on serait en peine d'en déterminer une ${ }^{24}$. C'est plutôt un assemblage des itinéraires que l'auteur a fréquentés.

22 . "Damascus drawn by J.-L. Porter 1855", dans Josias Leslie Porter, Five Years in Damascus, including an account of the history, topography..., Londres, J. Murray, 1855, fin du vol. 1.

${ }^{23}$. A. von Kremer, op. cit. Traduction d'un passage relatif à l'organisation de l'espace domestique, Les cahiers de la recherche architecturale $\mathrm{n}^{\circ} 10 / 11,1982, \mathrm{p} .112-114$.

${ }^{24}$. Par exemple, selon ce document, la takiya Sulaymâniyya serait située à mi-chemin entre la mosquée des Omeyyades et le village de Mazza alors qu'elle est en réalité localisée plus près de la mosquée, environ au tiers de la distance qui sépare la mosquée du village (1 400 mètres sur 4 400). 


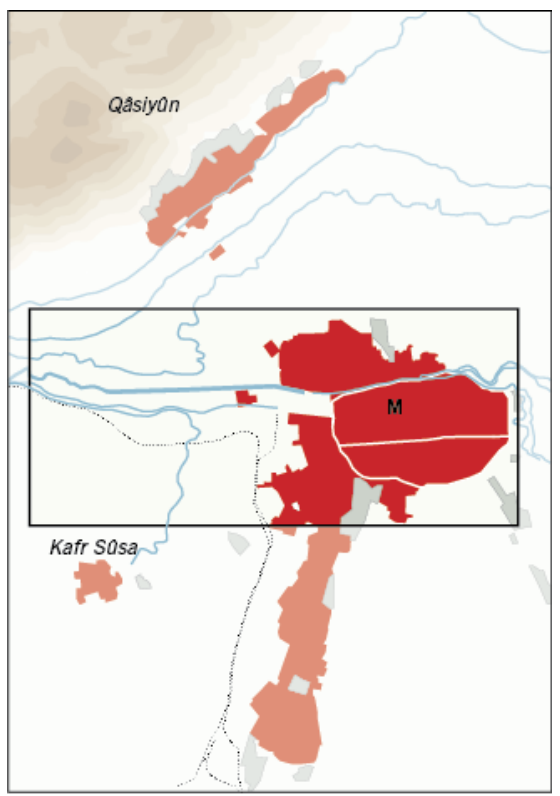

Fig. 5. Périmètre approximatif du plan de Alfred von Kremer. Pour localiser les édifices qui l'intéressent, von Kremer a adopté un périmètre très indépendant de celui de l'agglomération. Document établi par l'auteur.

Le long des rues, il mentionne les lieux qui lui semblent utiles pour se repérer dans la ville. Ce mode de composition, par compilation d'éléments linéaires, n'est pas suffisant pour constituer un plan. Il laisse d'importantes incertitudes et surtout, dans la mesure où les itinéraires se recoupent à plusieurs reprises, l'auteur n'identifie pas toujours les sections communes aux uns et aux autres. Ainsi, ce document comporte-t-il des incohérences qui interdisent de restituer l'ensemble des voies qu'il représente, notamment dans la zone située entre le quartier chrétien et la mosquée des Omeyyades.

La faiblesse des données topographiques de la carte de Kremer est compensée par les nombreuses désignations des lieux. Ceux qui sont assez vastes, tels que les quartiers et les cimetières, sont nommés directement sur le document tandis que les autres, en particulier les édifices, sont identifiés par un renvoi à une liste imprimée qui ne compte pas moins de cinquante-sept entrées. Le type des lieux qui y sont mentionnés exprime l'intérêt de l'auteur pour les points de repère civils. Plus de la moitié de la légende est consacrée aux portes de la ville, à ses voies de circulation (souks, rues et ruelles) et aux entrepôts ${ }^{25}$. Ce sont ensuite les mosquées qui sont les plus nombreuses, on en compte quinze. Il est enfin remarquable que Kremer ne repère pas un seul établissement voué au culte chrétien. $\mathrm{Ce}$ choix n'est pas habituel et l'auteur se démarque ainsi des autres voyageurs européens qui mentionnent plus volontiers les établissements de la minorité chrétienne que ceux de la communauté musulmane. De ce point de vue, Kremer fait oeuvre de savant plus que de voyageur ; son plan n'est pas destiné à guider d'éventuels successeurs mais, comme le titre de son livre le précise, à renseigner les lecteurs sur l'organisation spatiale de la ville. Il est par exemple remarquable que, pour indiquer la localisation de plusieurs mosquées situées au sud du quartier du Mîdân, il représente ce faubourg dans une vignette (fig. 5).

\section{Josias Leslie Porter (1855)}

Comme son prédécesseur, Josias Leslie Porter n'est pas cartographe. C'est un religieux anglais qui séjourne à Damas à la faveur d'un voyage de plusieurs années consacré aux ruines antiques de la région. Son plan de Damas est publié au cours de l'année 1855 avec la relation de ce voyage, il en illustre le chapitre qui traite de la topographie et des

\footnotetext{
25 . Khân en arabe.
} 


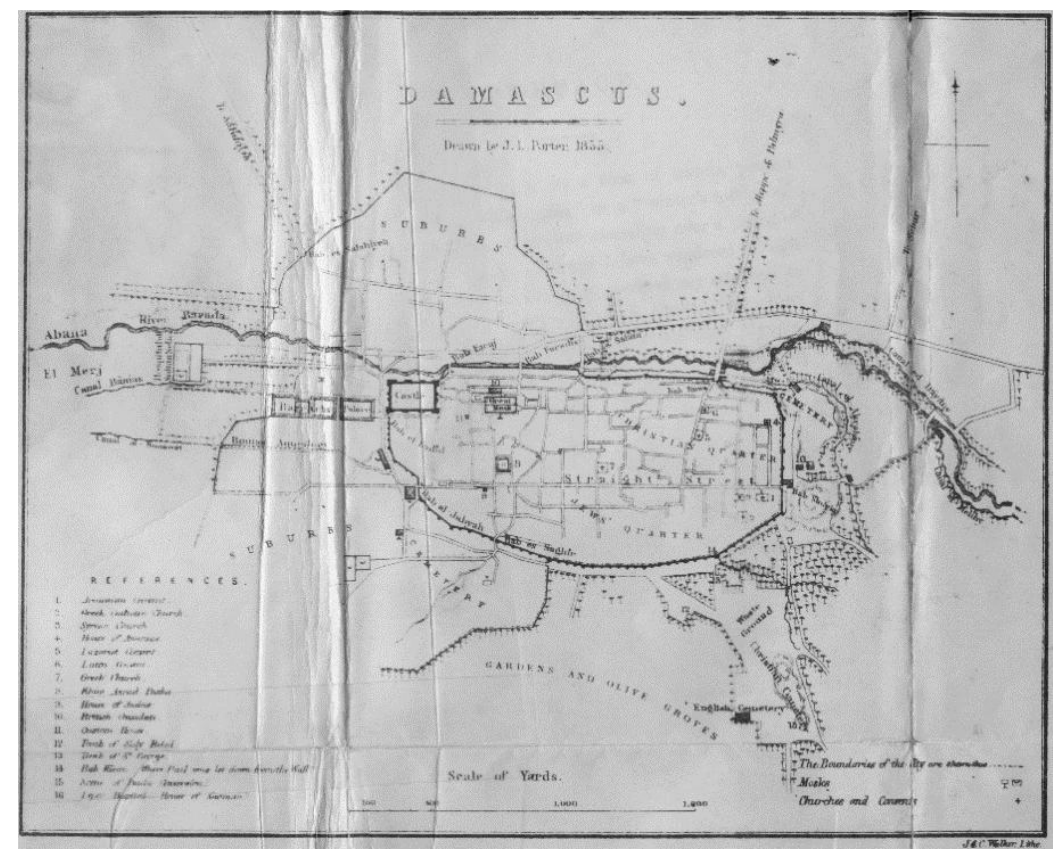

Fig. 6. Plan de Josias Leslie Porter. 1855. Le périmètre très aplati de la ville intra-muros témoigne du caractère approximatif du relevé. "Damascus Drawn by J.-L. Porter 1855" (voir note 22), reproduit avec l'autorisation de l'institut français d'études arabes de Damas.

antiquités de la ville (fig. 6). Comme en témoignent ses différences avec les précédents, ce plan ne résulte pas d'une copie mais d'un nouveau relevé. Alors que Kremer a été plus attentif aux itinéraires qu'aux territoires, Porter associe les deux types de données : un réseau dense de rues et des périmètres (enceinte et limites de l'urbanisation) représente une forme générale et des détails cohérents avec la topographie. De manière évidente, l'auteur dispose d'un savoir-faire plus élaboré que son prédécesseur. Cependant, les qualités géométriques de son plan ne résistent pas à un examen plus approfondi. Malgré son échelle graphique, les distances qu'il indique sont approximatives ${ }^{26}$. La répartition spatiale des erreurs montre que l'auteur n'a effectué aucune triangulation et qu'il a peu utilisé la boussole. Son évaluation des distances est aussi très sommaire. Par exemple, alors qu'il aurait pu utiliser la rue Droite et ses multiples carrefours comme base pour la construction de son plan, il en mesure seulement la longueur totale et place au jugé (avec d'importantes erreurs) les autres rues qui la croisent.

De la même manière que Niebuhr et Kremer, Porter ne prend pas en compte l'ensemble de l'agglomération. Il ne mentionne pas le faubourg de Sâlihiyya situé au nord de la ville tandis que la frange septentrionale du Mîdân est à peine esquissée (fig. 7). Par contre, les environs proches de l'est et de l'ouest ne manquent pas d'informations quant au réseau hydrographique, à l'occupation des sols et à la situation de plusieurs établissements. Ainsi, la région des cimetières chrétiens, située au sud-est de la ville, est-elle représentée avec des détails qui expriment l'attention de l'auteur à son égard. Le cimetière anglais y fait l'objet d'une mention particulière, C'est à travers sa légende que Porter manifeste le mieux ses intérêts. Sur les dix-huit entrées qui la composent, la moitié est consacrée à des lieux de culte chrétiens : églises et couvents en particulier; les autres renvoient à des établissements civils et administratifs. A l'inverse, à part pour la mosquée des Omeyyades,

26. Sur un échantillon de 19 mesures, l'erreur moyenne est de 20\%; 8 seulement sont inférieures à 10\%, 7 sont entre 10 et $25 \%$, et 4 sont supérieures à $25 \%$. 


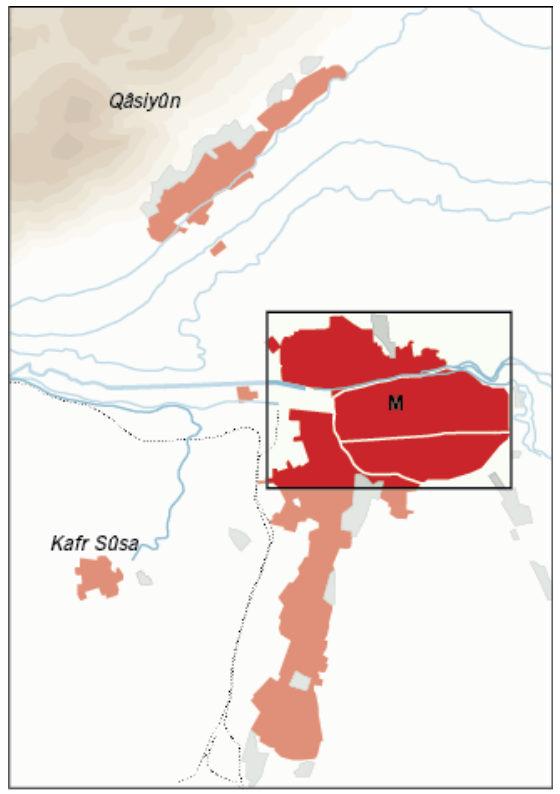

Fig. 7. Périmètre approximatif du plan de Josias Leslie Porter. Alors que la zone des cimetières située au sud-est de la ville est l'objet de plusieurs indications, le quartier du Mîdân, qui regroupe quelques dizaines de milliers d'habitants, semble ignoré par Porter. Document établi par l'auteur.

les lieux de culte musulmans et juifs ne sont jamais nommés ; les mosquées sont localisées par un figuré tandis que les synagogues semblent totalement absentes malgré l'indication d'un quartier juif. La légende de Porter ne concerne pas seulement les édifices, elle indique aussi la limite de l'urbanisation - boundaries of the City. Ce tracé correspond à celui de l'enceinte de manière partielle ; même s'il ne prend pas la peine de représenter l'ensemble des faubourgs et même s'il ne donne pas autant de détails pour les périphéries que pour le centre, l'auteur a compris que la mauvaise correspondance entre la limite de l'urbanisation et celle de l'enceinte était une particularité de l'organisation spatiale de Damas au milieu du dix-neuvième siècle.

Malgré son aspect schématique et d'importantes erreurs de proportion, le réseau viaire n'est pas négligé. Porter exclut les impasses et les rues secondaires mais il retient plus de vingt kilomètres de grandes voies de circulation. Leur restitution à partir d'un plan plus récent montre qu'elles résultent d'un relevé ${ }^{27}$. L'auteur semble avoir parcouru les vingt kilomètres représentés mais il n'en a pas consigné tous les détails. Au contraire, s'il a noté les directions principales et les points de départ et d'aboutissement de chaque section, il a fait peu de cas des changements de direction, des ressauts, des redents et des décalages qui caractérisent le réseau des rues de Damas. Plusieurs rues en biais ou celles qui sont constituées par de multiples coudes sont souvent simplifiées.

La restitution montre aussi que l'auteur n'a pas parcouru tous les quartiers avec la même attention. Tout d'abord, la ville intra-muros est plus détaillée que les faubourgs de Qanawât, 'Amâra et Sûq Sârûjâ dont Porter emprunte seulement les voies de sortie de la ville en négligeant le maillage du tissu environnant. Le long de ces voies, lorsqu'il repère une rue qui semble plus importante que les autres, il note son point de départ et représente une amorce pas toujours cohérente avec les détails des lieux. Hors les murs, sur la rive sud du Baradâ, le réseau relevé par l'auteur préfigure l'organisation des nouveaux quartiers de la seconde moitié du dix-neuvième siècle. Dans cette partie, occupée suivant une faible densité par des établissements publics ou religieux, et composée autour de rues qui semblent assez rectilignes, il était peut-être plus facile d'effectuer des relevés que dans les

27. Seulement trois courtes sections présentent une incohérence avec l'état des lieux, il s'agit dans deux cas de prolongements abusifs. 
faubourgs principalement occupés par de l'habitat. Enfin, à l'intérieur de l'enceinte, le réseau des rues n'est pas figuré de manière homogène. Le quartier chrétien et la rive nord de la section centrale de la rue Droite sont les plus complets tandis que le sud et le sudouest de la ville ne semblent pas avoir été visités. Porter y dessine, comme autant d'impasses, les voies qu'il a entrevues depuis la rue Droite sans chercher à savoir où elles aboutissent.

\section{L'archéologie et le périmètre des plans}

Comme le plan de Niebuhr, ceux de Kremer et de Porter représentent mal les faubourgs. Mais, alors que pour le premier (dont le savoir-faire n'est pas en cause), on peut imputer cet oubli à la courte durée de son séjour, les autres passent plusieurs mois, voire plusieurs années, à Damas. Ils ne pouvaient donc pas ignorer l'existence des faubourgs que leurs cartes ne mentionnent pas. Ces lacunes résultent donc de choix délibérés de la part des auteurs. L'un et l'autre, orientaliste et archéologue, publient des plans qui sont tout d'abord chargés d'accompagner leurs textes et d'indiquer la localisation des ruines, des édifices et des inscriptions dont ils traitent. Suivant cet objectif, on comprend mieux que les faubourgs peu pourvus en lieux d'intérêt archéologique aient été négligés.

\section{Seconde génération (1860-1924)}

Quoique dressé quelques années seulement après celui de Porter, il faut attendre vingt ans pour que le plan suivant soit publiée ${ }^{28}$. L'auteur, Emmanuel Guillaume Rey, est aussi archéologue mais son plan témoigne d'un tout autre point de vue. Il n'a pas pour vocation d'illustrer un texte et des descriptions, c'est plutôt un outil de travail et de repérage qui, sans a priori, représente l'ensemble de l'agglomération et de ses environs proches.

La qualité du plan de Rey n'a rien à voir avec celle des précédents. Plus tard, plusieurs publications comportent des plans de Damas cependant, chez E. Reclus en 1884, chez F. Schrader, cinq ans plus tard, ou encore en 1914 avec la carte de la Turquie, ces plans sont de petites vignettes qui figurent seulement, et de manière sommaire, le périmètre de la ville, quelques rues et guère plus de monuments ${ }^{29}$. Ils résultent de la simplification du précédent, ou, peut-être, d'un document intermédiaire dont les traces n'ont pas été retrouvées, mais en aucun cas d'un nouveau relevé. Ensuite, il faut attendre la seconde moitié des années 1910 pour que des cartographes s'intéressent à nouveau à Damas.

Durant la Première Guerre Mondiale, et à la faveur de l'occupation de la région par l'armée allemande, deux de ses officiers, Karl Wulzinger et Carl Watzinger, dressent les plans de plusieurs quartiers de Damas ${ }^{30}$. Avant qu'ils ne soient publiés par leurs auteurs, ces documents sont assemblés, complétés et imprimés par l'autorité publique ottomane en $1918^{31}$. Quelques années plus tard, au début des années 1920, l'administration mandataire

28 . "Plan de Damas et de ses faubourgs", dans Chauvet et Isambert, op. cit.

29 . "Damas", dans Elisée Reclus, Nouvelle géographie universelle, Paris, Hachette, 1884, livre 9, p. 789, fig. 142 ; "Damas", dans Franz Schrader, Ferdinand Prudent et Edouard Anthoine, Atlas de géographie moderne, Paris, Hachette, 1889, pl. 39 ; "Plan de Damas et de ses faubourgs", dans Carte de la Turquie d'Asie, Beyrouth, Service géographique de l'armée, 1914, en médaillon sur la feuille 7.

30 . Karl Wulzinger et Carl Watzinger, Damascus die Islamische stadt, Berlin, de Gruyter, 1924, vol. 2, pl. 57 , pl. 62 et p. 101.

31. Plan titré et légendé en langue arabe: Al-cham dimachq harita [plan de Damas], diwân al-chura al-(sic), ghurfa al-harita, [conseil consultatif (sic). bureau de dessin], nom de l'imprimerie illisible, 1337 [1918], une feuille, 1:10 000 . 
inaugure un cycle de production cartographique sans précédent. En vingt ans, elle dresse une série de documents à différentes échelles qui rendent compte de l'organisation de l'espace urbain depuis le découpage parcellaire (échelle 1:500) jusqu'aux grandes masses de l'urbanisation (échelle 1:50 000) (32 $^{32}$.

\section{Emmanuel Guillaume Rey (1882)}

Emmanuel Guillaume Rey, a laissé de nombreuses publications ${ }^{33}$. C'est un érudit qui travaille en histoire du Levant, durant la période médiévale en particulier. Il est membre de plusieurs sociétés savantes et effectue des missions en Syrie à la demande du Ministre de l'instruction publique à partir de la fin des années 1850 . Son premier séjour semble dater de 1857-1858, il travaille alors dans le Hauran et dans les environs de la mer Morte ${ }^{34}$. L'année suivante, en décembre, il étudie la topographie de la ville d'Acre au treizième siècle ${ }^{35}$; en 1864-1865, à l'occasion de son troisième séjour, il travaille dans le nord de la Syrie $^{36}$. Atteint de paludisme, il écourte cette mission dont il semble que ce soit la dernière. Les rapports de la première et de la dernière mission sont publiés, rédigés avec la plus grande minutie, ils indiquent ses itinéraires au jour le jour.

Rey passe par Damas dès son premier voyage ; il y séjourne un peu moins de deux semaines au cours desquelles il effectue de nombreuses visites et prépare son expédition vers la mer Morte ${ }^{37}$. Compte tenu de cet emploi du temps (dont il rapporte tous les détails) il est impensable que Rey ait alors pu dresser un plan de Damas. Le dernier voyage, consacré à la Syrie du nord, ne le conduit pas à Damas. C'est donc au cours de son séjour précédent qu'il a pu en relever le plan ; il n'a malheureusement pas laissé de rapport de cette mission $^{38}$. Suivant ces informations, les renseignements qui sont à l'origine du plan encarté dans le guide de 1882 auraient été réunis au cours de l'hiver 1859-1860. D'autres hypothèses sont envisageables. Lors de ses voyages, Rey fréquente assidûment les consulats français et les membres des multiples communautés religieuses alors protégées par la France. Il n'est pas impossible qu'il se soit procuré ce plan au cours d'une de ces occasions. Par exemple, au mois d'octobre 1857, alors qu'il est de passage à Saïda, il rencontre le docteur Gaillardot, ancien chirurgien dans l'armée égyptienne ${ }^{39}$. Celui-ci, tout autant épris d'érudition que Rey, se livre à de multiples observations et effectue aussi des relevés topographiques. Ses plans de Saïda et de Tyr, publiés par Renan au début des années 1870, témoignent de son talent

32 . Les principaux documents de cette période sont, par échelle croissante : Ville de Damas, Damas, Régie du cadastre, 1930-1934, 1:500 : Ville de Damas, Damas, Régie du cadastre, 1935, 1:2 000 ; Lucien Vibert, Plan de Damas, Damas, Ville de Damas, s.d. [1924], 1:5 000 ; Damas, s.l., Service géographique des Forces françaises libres au Levant, 1942, 1:10 000 ; Damas, Beyrouth, Institut géographique national au Levant, 1941, 1:20 000 ; Environs de Damas, Paris, Institut géographique national, 1941, 1:50 000.

33 . Catalogue général des livres imprimés de la bibliothèque nationale, vol. 150, Paris, Imprimerie Nationale, 1938, p. 45-47.

34 . Emmanuel Guillaume Rey, Voyage dans le Haouran aux bords de la mer Morte exécuté pendant les années 1857 et 1858, Paris, Arthus Bertrand, s.d. [1861], un vol. et un atlas.

35 . Emmanuel Guillaume Rey, Etude sur la topographie de la ville d'Acre au Xlle siècle, Paris, s.n., 1879.

36 . Emmanuel Guillaume Rey, Rapport sur une mission scientifique accomplie en 1864-1865 dans le nord de la Syrie, Paris, Imprimerie impériale, 1867.

37. Rey arrive à Damas le 26 octobre 1857 et en repart le 9 novembre suivant : E. G. Rey, Voyage..., op. cit., p. 17-38.

38 . Dans ses deux publications consacrées à Acre durant la période médiévale, Rey ne fait aucune allusion à un éventuel séjour à Damas ; E. G. Rey, Etude..., op. cit.; Emmanuel Guillaume Rey, Supplément à l'étude sur la topographie de la ville d'Acre au Xlle siècle, Paris, fascicule de 18 pages extrait des mémoires de la société nationale des antiquaires de France, tome XLIX, 1889.

39 . E. G. Rey, Voyage..., op. cit., p. 2. 
dans ce domaine ${ }^{40}$. Gaillardot dispose également de multiples notes sur le Hauran, apprenant que Rey doit y effectuer une mission, il lui transmet sa documentation et ses relevés. Dans ce contexte, on ne peut pas exclure que les renseignements à l'origine du plan de Damas ne soient peut-être pas de la main de Rey car, si pour le Hauran, la publication d'un rapport lui permet d'adresser ses remerciements à Gaillardot, le plan de Damas n'est accompagné d'aucune notice explicative qui lui aurait permis de rendre compte de ses sources.

Cette hypothèse est d'autant plus probable que c'est au complément de la zone Nord-ouest de sa carte que les notes de Gaillardot sont mises à profit par Rey; or, cette zone est justement celle qui représente les environs de Damas. Ainsi, le plan de Damas serait antérieur à 1857. Datation ante quem seulement que l'absence d'informations quant à la biographie de Gaillardot ne permet pas de préciser. Le contenu du plan et sa confrontation aux données archéologiques n'apporte pas d'éclairage supplémentaire ${ }^{41}$. Dernière hypothèse, peu probable, Rey s'est peut-être procuré son plan plus tard, en France. Dans ce cas, il peut être daté par les données archéologiques et serait antérieur à $1870^{42}$.

On ignore quelle est la formation de $\operatorname{Rey}^{43}$; suivant les relations de ses voyages, il s'intéresse plus aux sites antiques et médiévaux qu'à la période contemporaine, il ne traite pratiquement pas des villes qu'il visite et ses atlas n'en comportent pas le moindre plan. Lors de son troisième voyage, alors qu'il séjourne plus de trois mois à Beyrouth durant l'hiver il n'y attache pas le moindre intérêt dans ses publications. Suivant ces indications, on pourrait penser que si Rey a relevé un plan de Damas, il l'a fait à la mesure de l'intérêt qu'il semble porter à cette ville dans ses textes, c'est-à-dire de manière succincte. Or, le plan publié par Chauvet et lsambert correspond mal à cette définition, il est au contraire de très bonne qualité, et pour cause, Rey est spécialiste de relevés cartographiques. A l'issue de sa mission en Syrie du nord au milieu des années 1860, il publie une carte détaillée de la région qui rassemble l'ensemble des connaissances alors disponibles ${ }^{44}$. Les éditeurs de guides ne s'y trompent pas; Chauvet et Isambert ne font pas appel à Rey seulement pour le plan de Damas, il leur fournit aussi les renseignements nécessaires à l'établissement des plans d'Acre, de Nazareth, d'Antioche et d'Edesse (Urfa) ${ }^{45}$. S'ils sont moins bien documentés que celui de Damas, ils n'en expriment pas moins une maîtrise assurée de la cartographie urbaine. En outre, la carte de l'ensemble de la Syrie publiée dans le guide est aussi de la main de Rey. Ce document est assez nouveau pour que l'auteur consacre un opuscule à son élaboration ${ }^{46}$. Il y donne la liste de ses sources et expose ses méthodes avec une précision qui rend compte sans ambiguïté de ses compétences en la matière. En

40 . Ernest Renan, Mission de Phénicie dirigée par M. Ernest Renan, Paris, Imprimerie nationale, 1874, pl. LXVIII, LXIX et LXVI.

41 . Confrontation conduite à partir de l'inventaire dressé par D. Sack au cours des années 1880 ; Dorothée Sack, Damaskus. Entwicklung und Struktur einer orientalisch-islamischen Stadt, Mainz, Verlag Philipp von Zabern, 1989, p. $114-121$ et pl. 8.

42 . Ibid, repères numéros $5.2,5.5$ et 5.8 en particulier.

43 . Le fait que Rey ait démissionné de la société nationale des antiquaires de France avant son décès semble l'avoir privé de la faveur d'une notice nécrologique dans le bulletin de cette société : Bulletin de la société nationale des antiquaires de France, 1905, p. 340.

44 . E.G. Rey, Rapport..., op. cit.

45 . Chauvet et Isambert, op. cit. ; "Acre" : périmètre général des environs de la ville et des enceintes, pas de détail du tissu urbain, env. 1:20 000, p. 411; "Nazareth" : plan des principales rues, très déformé, environ 1:13 000, p. 439 ; "Antioche": plan des principales rues et du périmètre de l'enceinte, environ 1:30 000, p, 727 ; "Urfa" : plan très sommaire du réseau viaire et des environs de la ville, sans échelle, p. 747.

46. Emmanuel Guillaume Rey, Notice sur la carte de la Syrie, Paris, Hachette, 1885. 


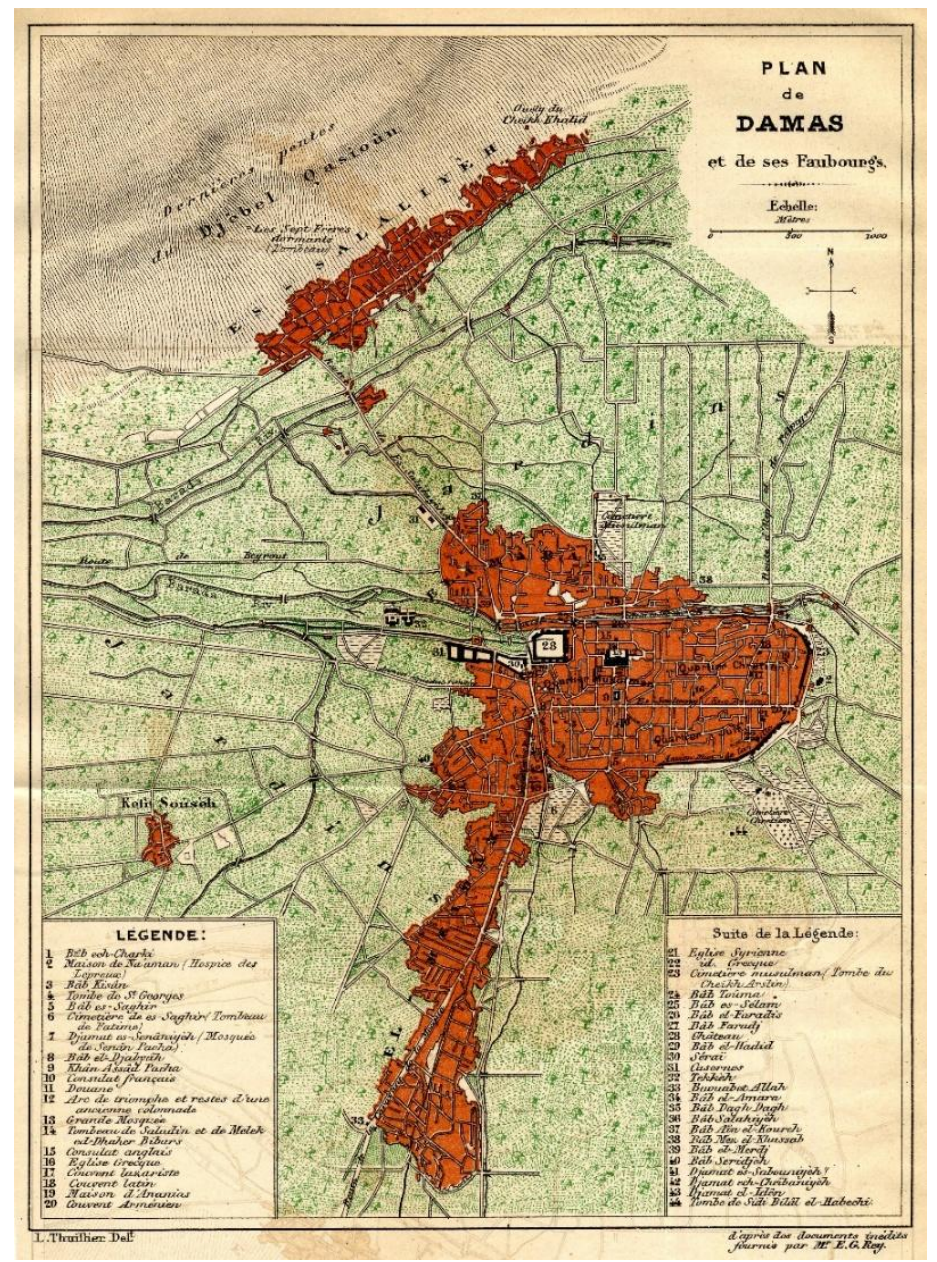

Fig. 8. Plan de de Emmanuel Guillaume Rey, 1860. Il s'agit du premier plan de Damas dressé suivant une base triangulée et qui représente l'ensemble de l'agglomération et de ses environs proches. "Plan de Damas et de ses faubourgs..." (voir note 3), reproduit avec l'autorisation de l'Institut français d'études arabes de Damas.

quelques pages, Rey révèle qu'il n'ignore rien des techniques de relevé topographique ni du principe des coordonnées astronomiques.

En ce qui concerne son plan de Damas, la précision des tracés indique que c'est à partir d'un document détaillé, dressé à une échelle voisine du 1:10 000 (c'est-à-dire dans un format d'environ 65 par 50 centimètres), que ce plan a été gravé. C'est en tout état de cause le résultat d'un long travail de terrain - plus de soixante-dix kilomètres de rues ont été mesurés - dont on peut estimer la durée à trois ou quatre semaines au minimum. Ce plan n'est donc pas un croquis réalisé à la faveur d'un désœuvrement, il résulte au contraire d'un projet ; l'auteur a décidé de dresser un relevé complet de Damas, il n'ignorait pas les difficultés afférentes à l'entreprise et il s'est donné les moyens de la conduire à son terme avec toute la qualité envisageable. Cet examen ne permet pas de trancher quant à la date exacte de relevé du plan publié en 1882. Cependant, Rey disposait des compétences pour effectuer un tel relevé ou pour le diriger. D'autre part, les exemples de Porter (qui n'est pas, rappelons-le, cartographe) et de Kremer qui, outre son plan, dresse aussi de nombreux dessins d'édifices, de ruines et de minarets et relève plusieurs dizaines d'inscriptions, montrent qu'il était possible d'arpenter les rues de Damas en mesurant des angles, en prenant des notes et en effectuant des relevés au milieu du dix-neuvième siècle. Enfin, il 


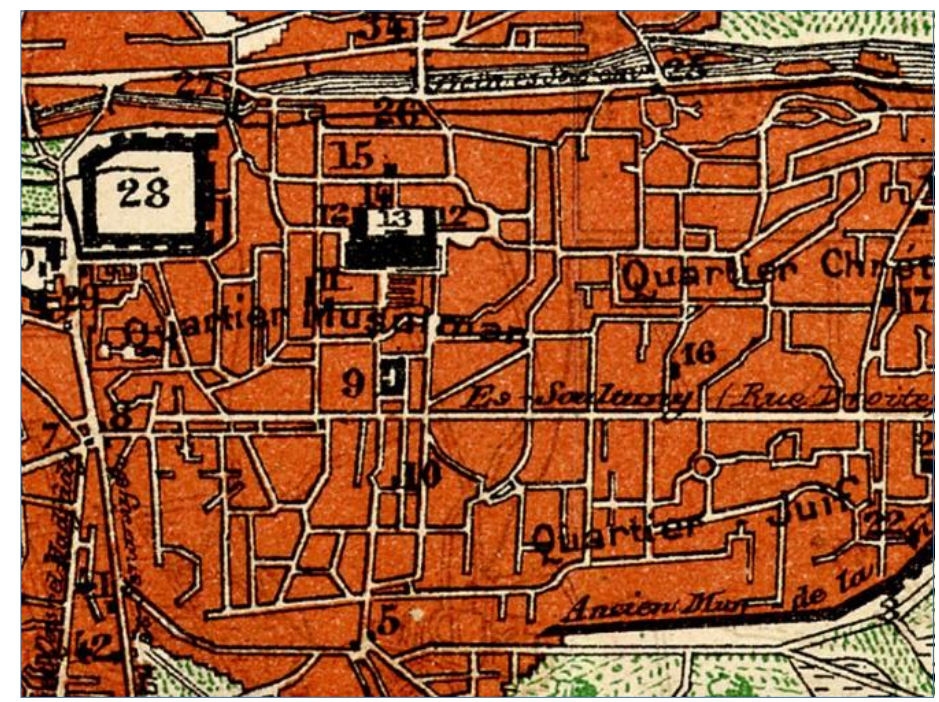

Fig. 9. Plan de Emmanuel Guillaume Rey, 1860, détail. Malgré sa complexité, le maillage du réseau des rues présente de manière générale des dispositions conformes aux relevés plus récents. Extrait de "Plan de Damas et de ses faubourgs" (voir note 3), reproduit avec l'autorisation de l'Institut français d'études arabes de Damas ; même cadrage que la fig. 13.

ne faut pas exclure que l'auteur a pu dissimuler son activité sous l'aspect de promenades ; les espions ne pratiquaient pas autrement ${ }^{47}$.

Le plan de Rey a été publié en 1882 dans un guide archéologique de l'Orient dirigé par A. Chauvet et E. Isambert. Dressé, gravé et imprimé à Paris par les services de l'éditeur du guide, il porte la mention "d'après les renseignements inédits fournis par Mr E. G. Rey" (fig. 8 et 9$)^{48}$. Malgré sa petite taille $(16$ par $22 \mathrm{~cm})$ et malgré l'échelle de sa reproduction (env. 1:33 000), la gravure de ce document est assez fine pour permettre d'en comparer les dispositions avec des plans plus récents. Celui publié en 1924 sous la direction de Lucien Vibert (voir plus bas) constitue une base sûre pour effectuer cette comparaison et pour construire la restitution géométrique du plan de Rey (fig. 10) ${ }^{49}$. Elle indique tout d'abord qu'il respecte les proportions relatives des différentes parties de la ville. Compte tenu de l'étendue représentée - plus de trente kilomètres carrés - et de la faible distorsion de l'ensemble, on peut même se demander si l'auteur n'a pas procédé à une triangulation générale depuis les flancs du mont Qâsyûn pour fixer la position relative des grandes masses de l'urbanisation ${ }^{50}$. La comparaison des distances entre une douzaine de points de repère confirme cette hypothèse ${ }^{51}$. Les détails du tissu urbain sont tout aussi fiables, leurs

\footnotetext{
47 . Sur l'espionnage cartographique au dix-neuvième siècle, voir en particulier Kapil Raj, "La construction de l'Empire de la géographie. L'Odyssée des arpenteurs de Sa Très Gracieuse Majesté, la reine Victoria en Asie centrale", Annales Histoire, Sciences Sociales n 52:5, 1997, p. 1153-1180.

48 . Chauvet et Isambert, op. cit.

49 . Le plan relevé par Lucien Vibert entre 1921 et 1924 est le premier document cartographique de Damas dressé dans le cadre de l'administration mandataire. A l'échelle 1:5000, il représente les moindres détails du réseau de la voirie. L. Vibert, op. cit.

${ }^{50}$. Compte tenu de la topographie de la ville, il est assez facile, à partir de deux points de vue situés aux extrémités de Sâlihiyya, de relever la position de plusieurs repères. La rue principale de Sâlihiyya, pratiquement rectiligne et donc d'une longueur facile à mesurer, constitue une base idéale pour une telle construction.

51 . Sur 21 mesures, l'erreur moyenne est de 6,1\% avec un maximum de $14 \% ; 10$ présentent une erreur inférieure à $5 \%, 6$ entre 5 et $10 \%$, et 5 ont une erreur supérieure à $10 \%$. La répartition géographique de ces erreurs montre que Rey n'a pas privilégié telle ou telle partie. Il ne néglige ni la zone urbanisée de manière continue ni les environs. Par exemple, le village de Kafr Sûsah n'a pas été positionné "au jugé", il est remarquable qu'il soit situé à une distance très exacte de la porte Al-Jâbiya.
} 


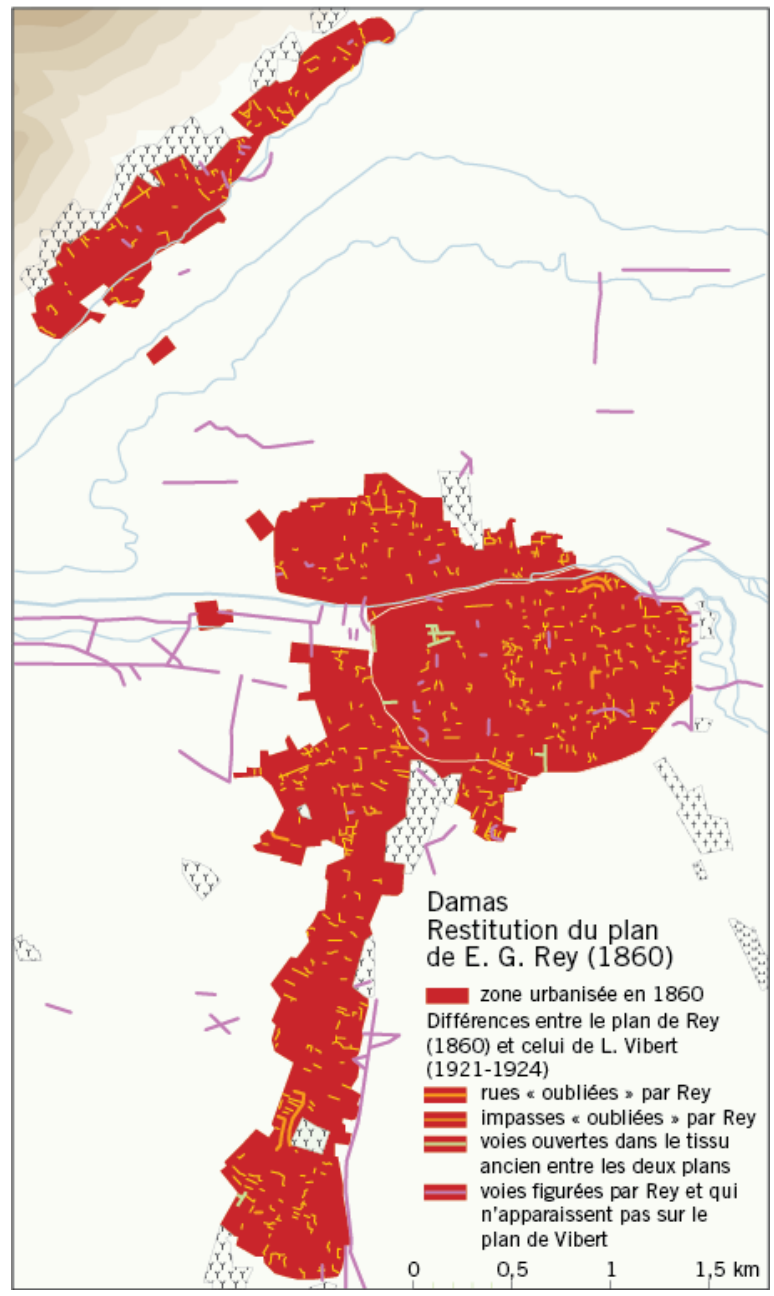

Fig. 10. Restitution du plan de Emmanuel Guillaume Rey. L'inventaire des différences entre le plan de Rey et celui de Lucien Vibert (voir fig. 17) confirme la qualité du premier ; seules les impasses les plus courtes ne sont pas représentées. Document établi par l'auteur d'après "Plan de Damas et de ses faubourgs" (voir note 3) et Vibert, Plan de Damas (voir note 32).

concordances montrent que ce plan a été dressé par deux relevés complémentaires : celui du périmètre de la ville d'une part, pris depuis l'extérieur, celui du réseau de la voirie et des monuments d'autre part, depuis l'intérieur. Comme en témoigne le caractère angulaire des tracés, ces relevés semblent avoir été faits au pas et à la boussole ${ }^{52}$ c'est-à-dire la seule méthode dont l'usage était possible pour un européen dans une ville de l'empire ottoman à ce moment-là.

A la fois à cause des moyens disponibles (il est nécessaire de parcourir chaque rue) et pour ne pas provoquer la suspicion de la population, les relevés des villes du ProcheOrient, établis par des européens au milieu du dix-neuvième siècle, se limitaient le plus souvent aux rues principales, les autres étaient omises ou bien elles étaient l'objet d'une figuration chargée de remplir le vide. Le plan de Rey est tout autre, l'ensemble des rues représentées a été relevé (sauf quelques rares exceptions) et les réseaux d'impasses explorées jusque dans leurs ramifications les plus fines. Cependant, comme dans de nombreux documents anciens, la précision n'est pas homogène, la zone nord-est de

52. L'exemple de la position de Kafr Sûsah le confirme. Sur cette méthode, voir Henri Seignobosc, Cours de topographie générale, Paris, Charles-Lavauzelle et Cie, $15^{\mathrm{e}}$ éd., 1951. 
Sâlihiyya semble avoir été relevée de manière plus rapide que les autres ; les distorsions y sont importantes et, en plusieurs lieux, on retrouve difficilement les éléments secondaires du réseau viaire. Par contre, le village de Kafr Sûsah, situé à quelques kilomètres à l'ouest de la ville, est figuré par un réseau conforme à la réalité. Les environs non urbanisés de l'agglomération ont aussi été l'objet d'attentions, les tracés des routes, des chemins et des canaux sont, de manière générale, cohérents avec ceux indiqués par les documents plus récents $^{53}$. On note une seule erreur explicite, il s'agit de la position du tombeau des Sept frères. Alors qu'il est situé en corniche au-dessus du Baradâ à environ trois kilomètres à l'ouest de la place Marja, le plan le représente dans le prolongement de la route de Sâlihiyya. Compte tenu de la précision générale de l'ensemble et du fait que la position exacte du tombeau serait en dehors du périmètre du plan, il est probable que cette erreur résulte d'un ajout de l'éditeur du guide plutôt que de l'auteur.

Malgré toutes ces qualités, la restitution géométrique de la voirie dessinée par Rey révèle plusieurs différences avec le plan de 1924. Tout d'abord, de nombreux éléments du réseau viaire de 1924 n'apparaissent pas chez Rey. Les manques localisés à l'intérieur de la zone construite sont les plus difficiles à interpréter. Soit il s'agit de transformations du tissu urbain, soit il s'agit d'oublis. Dans la plupart des cas, le caractère systématique du dessin permet de faire la part des choses. Le plan de Rey omet de nombreuses impasses mais pas n'importe lesquelles ; il s'agit de celles dont la profondeur n'excède pas une soixantaine de mètres. Ces manques ne sont pas des erreurs, ils semblent plutôt ressortir aux contraintes inhérentes à la cartographie.

Compte tenu de l'échelle de son plan, l'auteur (ou le graveur de la planche imprimée) n'était pas en mesure de dessiner l'ensemble du réseau viaire. Il a donc dû sélectionner parmi les données celles qui pouvaient être éliminées sans nuire à la compréhension de l'ensemble. Les impasses les plus courtes correspondent bien à cette définition. En outre, le fait que l'auteur représente les grands réseaux d'impasses témoigne du raisonnement qui a déterminé ses choix. On note cependant des écarts à cette règle dans les quartiers périphériques; les impasses oubliées y sont plus nombreuses et elles sont plus profondes. A Sâlihiyya et à Suwayqa, elles peuvent atteindre cent à cent cinquante mètres tandis qu'à Châghür et à Sûq Sârûjâ, l'auteur a oublié des réseaux d'impasses situés dans les deux cas à la frange de la zone agglomérée. Certaines impasses non figurées par Rey peuvent aussi correspondre à des voies ouvertes entre 1860 et 1924. En effet, la ville compte de nombreux jardins qui peuvent avoir été lotis durant cette période autour de nouvelles impasses comme c'est le cas pour les jardins des environs immédiats de la ville, au cours de la même période, dans le quartier 'Amâra al-barrâniyya en particulier.

D'autres manques, peu nombreux, ne concernent pas des impasses mais des rues ; là encore, il s'agit soit d'oublis, soit de transformations du tissu urbain. L'examen du plan parcellaire permet de trancher entre ces deux hypothèses ${ }^{54}$. On distingue ainsi des percements du tissu ancien et, en creux, les rues vraiment oubliées par Rey. On en compte seulement neuf dont quatre, les plus longues, sont situées loin du centre ${ }^{55}$. Enfin, Rey représente aussi quelques rues dont le plan de 1924 ne porte aucune trace. Alors que les différences de cet ordre sont souvent nombreuses dans les plans des villes de l'orient arabe

\footnotetext{
53 . Damas, 1:10 000, 1942, op. cit.

${ }^{54}$. Le plus souvent, les rues créées entre les deux relevés (1860 et 1930) sont plus larges et plus rectilignes que les autres.

55 . Ces rues appartiennent au réseau primaire de la voirie. On en compte deux dans le quartier Al-jûra et deux au Mîdân qui mesurent entre 200 et 350 mètres ; la plus longue présente une entrée dont l'étroitesse (environ un mètre) ne facilite pas le repérage. La longueur des cinq autres rues oubliées varie entre 60 et 120 mètres.
} 
des dix-huitième et dix-neuvième siècles, celui de Rey n'en comporte pratiquement pas à l'intérieur du périmètre urbanisé ${ }^{56}$. Il s'agit le plus souvent de tracés prolongés comme si le crayon (ou le burin du graveur) avait filé un peu trop loin, Seule exception à cette règle, une rue tracée dans le quartier israélite qui ne correspond à rien en $1924^{57}$.

Hors de la zone agglomérée, les chemins disparus entre 1860 et 1924 sont plus nombreux; ils sont regroupés pour la plupart dans les quartiers Baramika et Marja, c'est-àdire là où les travaux d'édilité et d'urbanisation ont été les plus abondants. En effet, Damas connaît à partir des années 1890 un important développement vers l'ouest. A la suite de la régularisation et de la rivière Baradâ, plusieurs établissements publics sont construits autour de la place Marja. Ils sont accompagnés par le terminus de la première voie ferrée vers le sud et vers Beyrouth - mise en service au milieu des années 1890. Plus tard, au début du vingtième siècle, la gare du chemin de fer de La Mecque (voie du pèlerinage) contribue aussi à favoriser l'urbanisation de ce quartier ${ }^{58}$. Dans cette situation, les décalages entre le plan de Rey et celui de 1924 dans le secteur ouest de la ville ne doivent pas être considérés comme des erreurs mais comme des témoignages d'un état antérieur disparu à la faveur de l'extension de la ville. Enfin, de part et d'autre du Mîdân, en dehors de la zone urbanisée, la représentation des chemins s'interrompt assez rapidement, le vide est alors rempli par un effet de gravure. Cette disposition montre que le document original était incomplet dans cette partie comme s'il n'avait pas été prévu de le publier dans ce format ou suivant ce périmètre.

Le plan de Rey compte peu de désignations mais elles sont choisies de manière judicieuse pour permettre aux voyageurs de se repérer, pour entrer et sortir de la ville (route de Beyrouth, route de Salahiyèh, route d'Alep et de Palmyre, route de la caravane de La Mecque), pour s'orienter dans les trois faubourgs principaux (el-Salaliyèh, eI-'Amâra, elMeidan) et dans la répartition générale des communautés religieuses (quartier musulman, quartier chrétien, quartier juif) ${ }^{59}$. Le nom d'une seule rue est indiqué, c'est celle qui traverse la ville de manière rectiligne d'un bout à l'autre : il s'agit d'un point de repère et d'un axe majeur. Pour éviter d'éventuelles confusions, l'auteur donne les deux désignations de cette rue (es-Saltany et rue Droite). Si la toponymie est peu abondante, la liste des renvois compte quarante-quatre entrées. L'ordre adopté dans cette liste est topographique dans ses deux premiers tiers, elle traite ensuite des environs de la ville suivant un ordre plus aléatoire. Cet "itinéraire" part de la porte orientale (Bâb charqî) et se termine à une mosquée située non loin de là, dans les environs Sud de l'agglomération. Les lieux indiqués sont concentrés dans la zone intra-muros et ses environs proches, le Mîdân en comporte un seul et Sâlihiyya aucun.

Cette légende a pour objectif principal d'indiquer des points de repère puisque ses deux cinquièmes sont consacrés à des portes (bâb ou baouabet) qui ne présentent aucun autre intérêt archéologique, civil ou religieux. Ce sont ensuite les lieux de culte musulman qui sont les plus nombreux, ils sont dispersés dans la liste. Si plusieurs mosquées et tombeaux ont un intérêt archéologique suffisant pour que les auteurs du guide leur consacrent une notice, ceux qui ne sont pas mentionnés dans le texte peuvent, au même

56 . On en compte une douzaine seulement.

57 . Il est aussi remarquable que, dans cette partie de la ville, les impasses "oubliées" sont un peu plus nombreuses que dans les autres quartiers intra-muros.

58 . Pour l'urbanisation de ce quartier, voir Stephan Weber, "Des Marga-Platz in Damaskus", Damaszener Mitteilungen $\mathrm{n}^{\circ} 10,1998$, p. 291-344.

59. Ces désignations ne sont pas des noms de quartier proprement dits mais l'indication de communautés religieuses majoritaires. 
titre que les portes, être considérés comme des points de repère. Viennent ensuite les établissements civils, militaires et archéologiques non religieux. Cette catégorie est la plus disparate, elle comporte aussi des points de repère tels que les casernes et une seule indication explicitement archéologique : un arc de triomphe et les vestiges d'une colonnade situés à proximité de la mosquée des Omeyyades. Les lieux de culte chrétien mentionnés par le plan sont les moins nombreux, ils sont tous localisés dans la moitié orientale de la ville. Enfin, il est remarquable que les établissements du culte israélite ne soient pas indiqués. La répartition des lieux entre les désignations inscrites sur le plan et la liste des renvois ne correspond pas à une hiérarchie mais à une logique de cartographe. La toponymie est réservée aux éléments linéaires - rues, enceintes ou aqueducs - et les lieux - quartiers ou cimetières - dont l'étendue est suffisante pour contenir les désignations. Elle est complétée par des renvois qui se rapportent aux éléments plus ponctuels. Une exception confirme ce principe ; deux tombeaux situés au nord de Sâlihiyya, dans une zone où il est possible d'inscrire leurs noms sans interférence avec le dessin, sont repérés directement sur le plan.

Le plan de Rey offre une dernière particularité, au contraire de tous ceux qui ont été publiés dans les guides plus récents. Il représente l'ensemble de l'agglomération damascène de telle manière que son périmètre dépasse largement celui où sont circonscrits les lieux dont il est question dans le texte. Ce plan permet d'effectuer des visites, voire des repérages, dans un périmètre étendu. Ce n'est donc pas seulement un guide, c'est aussi un outil comme le manifeste l'abondance des points de repère éloignés de la zone de visite conventionnelle de la ville. On l'a vu à partir de deux exemples, Rey ne semble pas très attentif aux dispositions du quartier israélite. Au contraire, on peut se demander s'il n'a pas mieux figuré les quartiers de prédilection des chrétiens que les autres tout simplement parce qu'il était sans doute logé dans ces quartiers et parce qu'il lui a été plus aisé d'y effectuer ses relevés ou encore parce qu'il a pu s'y faire guider plus facilement. Les deux zones de concentration des chrétiens sont alors Mîdân Tahtani et Bâb Tûmâ. Or, la cartographie de ces deux quartiers ne présente pas de différences avec ceux où résident les musulmans. Les impasses "oubliées" y sont aussi nombreuses, une petite rue a même été omise à Bâb Tûmâ.

Ainsi, le plan de Rey peut être considéré comme la première représentation de Damas suivant un mode de projection parallèle vertical qui en fait un véritable plan de la ville. A ce titre et au-delà de son intérêt pour l'histoire de la cartographie, il constitue une base fiable pour évaluer les mouvements de l'urbanisation durant la seconde moitié du dixneuvième siècle. En outre, ce plan révèle déjà une tendance à l'extension de l'urbanisation vers l'ouest qui préfigure celle de la fin du siècle. Le fait qu'il représente une situation antérieure à l'ouverture de la route carrossable entre Beyrouth et Damas montre que les promoteurs du développement de la ville n'ont pas attendu cet équipement pour investir de manière massive dans ses environs occidentaux. En 1860, outre le couvent des derviches (repère 32) qui date du début du seizième siècle, on trouve aussi dans ce quartier un palais (repère 30) et plusieurs casernes (repères 31 ) construites pour loger l'armée de métier mise en place à la suite du démantèlement des milices des janissaires par le sultan Mahmud II en 1836.

\section{Karl Wulzinger et Carl Watzinger [c.1917]}

Au cours de la Première Guerre Mondiale, Damas est occupée par l'armée du IIe Reich. A la faveur de leur présence et du pouvoir qui leur est conféré, deux officiers allemands épris d'archéologie dressent un recensement des édifices remarquables, des ruines et des inscriptions de Damas. Pour effectuer ce travail et pour localiser leurs 


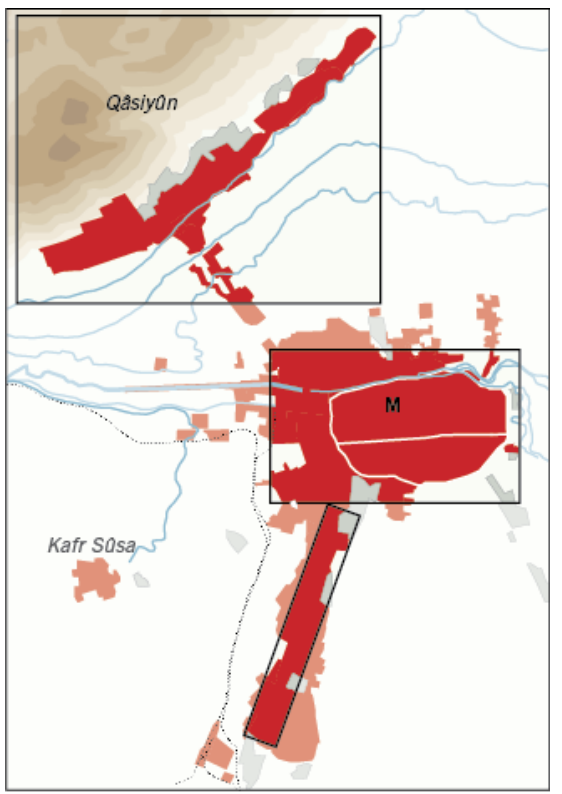

Fig. 11. Périmètres des plans de Karl Wulzinger et Carl Watzinger, relevés vers 1917. Les zones dépourvues d'édifices remarquables ont été négligées par les auteurs. Ainsi, les trois périmètres, représentés à des échelles différentes, ne sont pas contigus. Document établi par l'auteur.

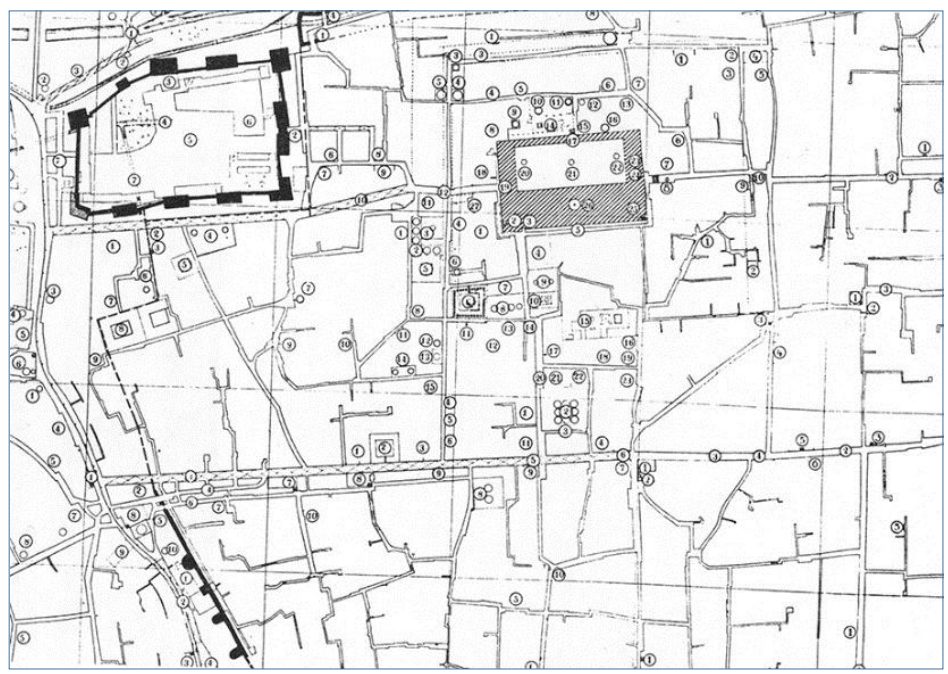

Fig. 12. Points de repère et la restitution du tracé de l'enceinte pour les sections dont les vestiges ont disparus, Extrait de "Plan der alt Stadt von Damaskus" (voir note 60), reproduit avec l'autorisation de l'Institut français d'études arabes de Damas ; même cadrage que les fig. 14 et 17 .

trouvailles, ils dressent trois plans partiels de la ville correspondants à son centre, au faubourg de Sâlihiyya et à celui de Mîdân (fig. 11 et 12$)^{60}$. Comme ceux de Kremer et de Porter, ces documents constituent les annexes d'un inventaire archéologique. Ils sont quadrillés et ponctués de plusieurs dizaines de points de repère qui renvoient à des notices descriptives. Cet usage, qui privilégie un point de vue local et nécessite une grande échelle de reproduction, explique le découpage de la ville en trois parties et leur représentation suivant trois échelles différentes en fonction de la densité des lieux à repérer : le centre est à l'échelle 1:4 000, le faubourg de Sâlihiyya est au 1:16 600 environ et celui de Mîdân au $1: 10500$.

60 . "Es-Salihije-Damaskus Nord (D. N.)", 1:16 660 ; "Plan der alt Stadt von Damaskus", 1:4 000 ; sans titre (plan du tracé de la rue principale du Mîdân), 1:10 500 ; dans K. Wulzinger et C. Watzinger, op. cit., vol. 2, pl. S7, p1. 62 et p. 101. 
Ces trois plans sont de qualités très différentes. Elles témoignent de l'attention inégale portée à leur réalisation par les auteurs. Celui du centre est le plus grand et le plus détaillé. Il indique l'ensemble du réseau de la voirie dans ses moindres ramifications, suivant ses ressauts et ses redents les plus fins et en respectant assez bien la largeur relative des voies. C'est aussi le plus précis; sa confrontation avec un document récent révèle une erreur moyenne à peine supérieure à un pour cent ${ }^{61}$. De toute évidence, ce plan résulte d'un relevé effectué suivant les règles de l'art. Un canevas trigonométrique semble avoir présidé à la construction de l'ensemble, les rues ont été arpentées et les angles mesurés avec précision,

Les deux autres plans représentent les faubourgs Nord et Sud de l'agglomération. Au contraire de ce que laisse entendre cette répartition géographique, il ne s'agit pas d'un même plan découpé en trois feuilles pour des raisons éditoriales mais de trois documents relevés de manière indépendante. La piètre qualité des représentations de Mîdân et de Sâlihiyya le confirme. Ces documents constituent des annexes de la publication plutôt que des extensions du plan du centre. Celui du Midân est le plus sommaire, il est limité au tracé de la rue principale de ce quartier et des amorces de celles qui la croisent. Celui de Sâlihiyya semble plus complet mais il n'est pas possible de restituer plus de la moitié des rues qu'il figure, c'est-à-dire les voies principales et quelques secondaires ; les autres, bien qu'elles ne présentent pas d'aberrations majeures par rapport à la structure générale du réseau, ne peuvent pas être identifiées sans de fortes incertitudes. La toponymie est peu abondante et sa répartition entre les trois documents est inégale.

Alors que le plan du centre ne comporte aucune désignation, ceux de Sâlihiyya et du Mîdân en comptent quelques-unes, comme si leur mauvaise qualité topographique devait être compensée par des indications toponymiques.

\section{Le plan de 1918 (fig. 13)}

Le plan daté de 1918 représente l'ensemble de la ville de Damas. Ce document, légendé en langue arabe, a été dressé par l'autorité publique ottomane à la demande du conseil consultatif (dîwân al-chûra) par son bureau de dessin (ghurfa al-harita) et imprimé en $1918^{62}$. Son niveau de détail est comparable à celui de Rey ; il figure le réseau viaire de la zone urbanisée ainsi que les routes et les chemins des environs, les limites de l'agglomération, celles des cimetières et de nombreuses désignations. Bien qu'il ait été relevé plus d'un demi-siècle après celui de Rey, ses proportions sont moins fiables et les erreurs plus diffuses ${ }^{63}$. Dans certains quartiers, à Mîdân en particulier, les distances sont parfois si mal proportionnées ${ }^{64}$ que l'on peut se demander si l'auteur n'a pas procédé par estimations plutôt que par des mesures. Cette faible fiabilité est d'autant plus étonnante que son échelle est assez grande (1:10 000) pour représenter le tissu urbain dans ses moindres

61. Sur 24 mesures, l'erreur maximum enregistrée atteint 4,2\%, la moyenne est de 1,2\%.

62. La précision qui suit la mention du conseil consultatif (diwân aI-chûra) est aI-harbî [de la guerre] ou aljuz'î [partiel (sic)]. Or, au moins deux conseils, connus sous des noms différents de ceux-ci siègent alors à Damas : le conseil municipal (majlis al-baladiyya) et le conseil public de la province (majlis al-'umumi). En l'état actuel des connaissances il n'est pas possible d'identifier à quelle instance la désignation mentionnée sur le plan correspond. Max L. Gross, Ottoman Rule in the Province of Damascus 1860-1909, Ph. D. dissertation, Georgtown University, 1979, p. 136 sq.

63 . L'erreur maximum enregistrée chez Rey est de $14 \%$, elle est de $27 \%$ pour le plan de 1918 ; l'erreur moyenne calculée sur 21 mesures prises depuis les mêmes points de repère est de $6,1 \%$ pour le plan de Rey et de 7,1\% pour celui de 1918. Enfin, on ne note aucune corrélation entre les deux séries d'erreurs.

64. Dans le quartier de Mîdân par exemple, le plan de 1918 donne 1350 mètres pour une distance de 1060 mètres, soit une erreur de $27 \%$. 


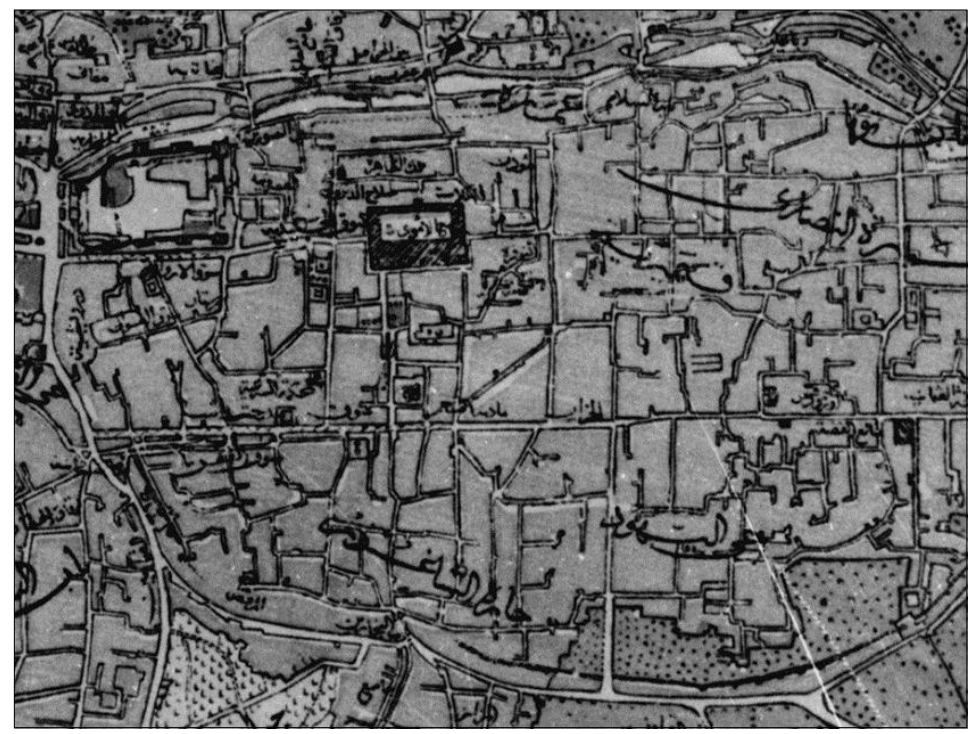

Fig. 13. Plan de 1918, détail. Ce plan, copie de ceux de Wulzinger et Watzinger pour le réseau viaire, comporte aussi de nouvelles indications, en particulier une abondante toponymie en langue arabe. Extrait de AI-cham dimachq... (voir note 31), reproduit avec l'autorisation de l'Institut français d'études arabes de Damas ; même cadrage que la fig. 9

détails. La restitution de ces tracés montre que l'attention de l'auteur n'a pas été identique dans tous les quartiers et qu'il a réparti ses moyens en fonction de leur éloignement du centre $^{65}$. Ainsi, dans la ville intra-muros, l'organisation du réseau viaire est pratiquement parfaite, seulement quelques impasses ont été oubliées. Plus on s'éloigne de ce centre, plus le relevé semble négligé. Pour l'extrémité du faubourg Sud, située à plus de trois kilomètres de la grande mosquée, le plan est encore moins fiable qu'à Sâlihiyya. Là, seulement quelques rues principales ont été relevées et leur représentation est simplifiée à l'extrême ; elles sont tracées en ligne droite entre leur point de départ et celui d'arrivée, indépendamment de leurs multiples détours. Pour les rues secondaires et les impasses, il est plus difficile de trancher car s'il n'est pas possible d'en restituer le maillage, l'exactitude de la position de plusieurs sections indique cependant qu'elles n'ont pas été tout à fait négligées. L'auteur ne les a pas parcourues - sinon il n'aurait pas transformé autant d'impasses en rues - il a seulement relevé la position des carrefours le long des routes principales. Puis, pour compléter son plan, il a raccordé les amorces ainsi définies entre elles. Il en résulte que de nombreuses impasses sont reliées et figurent un tissu plus fortement maillé qu'il ne l'est en réalité. L'approximation de ces méthodes se ressent dans la manière dont l'auteur trace le périmètre de l'urbanisation. Plusieurs sections n'en sont pas relevées mais déduites du tracé des rues comme en témoigne un "oubli" sur la frange orientale du Mîdân ${ }^{66}$.

65. Trois échantillons d'une cinquantaine d'hectares chacun ont été retenus pour tester la validité du plan de 1918 par restitution du réseau viaire. Le premier est situé à proximité du centre de la ville, à l'intérieur des murs, il comprend la citadelle, la mosquée des Omeyyades, le quartier Sidî Hamûd et, au sud de la rue Droite, une partie du quartier Châghûr ; le second est situé dans les faubourgs Nord, il représente l'extrémité de la route de Sâlihiyya et le quartier homonyme ; le dernier est le plus éloigné du centre, il représente la frange Sud du Mîdân. ${ }^{66}$. Dans ce quartier, une zone urbanisée en 1860, et aussi en 1924, n'est pas représentée en 1918, or il est peu probable que le tissu ait connu une récession puis une extension suivant des périmètres similaires durant la période considérée. 


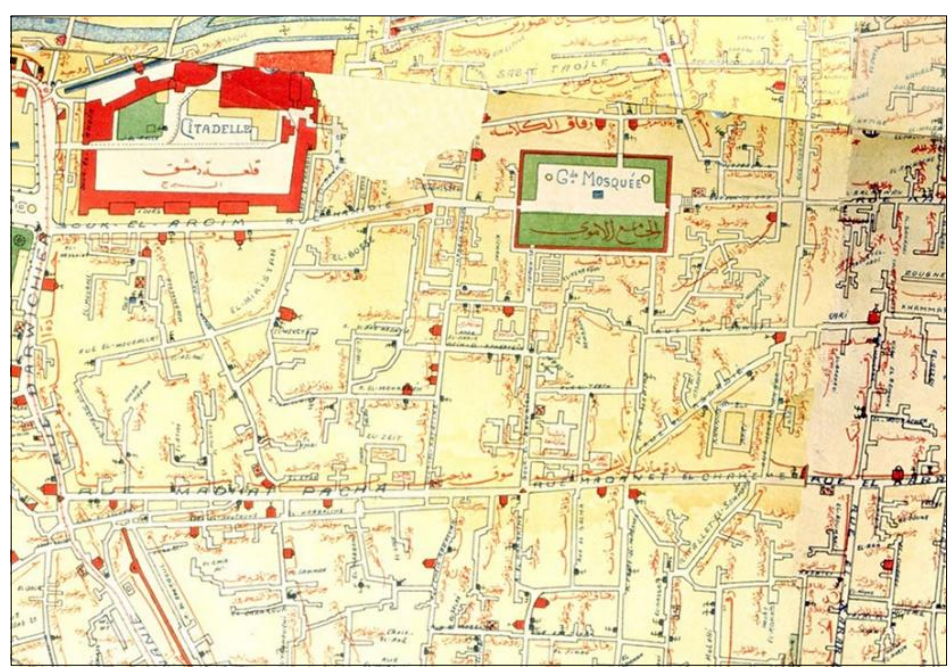

Fig. 14. Plan de Ahmed Rassem, 1922, détail, Ce plan est le premier qui représente sans exception l'ensemble du réseau viaire, jusqu'aux impasses les plus étroites. Sa toponymie, bilingue, est toute aussi exhaustive. Extrait de Harita churtâ dimachq (voir note 68), feuille 4, reproduit à l'échelle 1:10 000 avec l'autorisation de l'Institut français d'études arabes de Damas : même cadrage que les fig.12 et 17.

Cette hétérogénéité indique que l'auteur a compilé plusieurs sources. On ignore quelle était sa formation et de quel matériel il disposait. Il semble cependant évident qu'il

n'avait pas connaissance des principes élémentaires de la triangulation. Alors que ces principes nécessitent de procéder par division du territoire, en fixant d'abord les grandes lignes puis les détails de plus en plus fins, l'auteur du plan de 1918 a construit sa représentation par l'addition de relevés locaux sans prendre le soin de les "caler" dans une structure générale. En plusieurs points, ce document ressemble étrangement à ceux dressés par Wulzinger et Watzinger. Leur confrontation avec un plan plus récent montre que celui de 1918 résulte en fait de l'assemblage, dans une structure assez approximative, des trois plans dressés par les officiers Allemands ${ }^{67}$. Alors qu'ils sont publiés en 1924 et que l'armée allemande quitte Damas en octobre 1918, des copies de ces documents devaient circuler dans les bureaux de l'administration ottomane avant cette date.

Les données topographiques sont complétées par une abondante toponymie. Elle indique les noms des quartiers et ceux de nombreuses rues mais sa répartition géographique est hétérogène. Alors qu'à Sûq Sârûjâ, pratiquement toutes les rues et de nombreuses impasses sont nommées, on n'en compte aucune à Sâlihiyya et seulement quelques-unes dans les autres quartiers. L'arbitraire suivant lequel elles semblent choisies révèle que la toponymie n'a pas un objectif défini a priori mais qu'elle résulte plutôt d'un habillage qui rend compte des connaissances personnelles de l'auteur ou des opportunités qu'il a saisies. Là encore, l'auteur a compilé des informations partielles et hétérogènes.

67 . Pour la zone centrale, le plan de Wulzinger et Watzinger présente une erreur moyenne de 1,2\% (12 points de repère, 24 mesures), suivant les mêmes points de repère et les mêmes vecteurs, l'erreur moyenne de celui de 1918 est semblable. En outre, la confrontation des deux séries d'erreurs indique une forte corrélation $(0,88)$; pour Sâlihiyya, l'erreur moyenne du plan de Wulzinger et Watzinger est de 3,9\% ( 8 points de repère, 12 mesures), elle est de $4,2 \%$ pour celui de 1918 et la corrélation entre les deux séries est semblable à celle calculée pour la zone centrale. Enfin, pour les zones non couvertes par Wulzinger et Watzinger l'erreur moyenne du plan de 1918 atteint 5,5\% (11 points de repère, 16 mesures). Ce dernier chiffre confirme que les plans des Allemands n'ont pas été copiés sur celui publié en 1918 mais que c'est le contraire qui a eu lieu. 


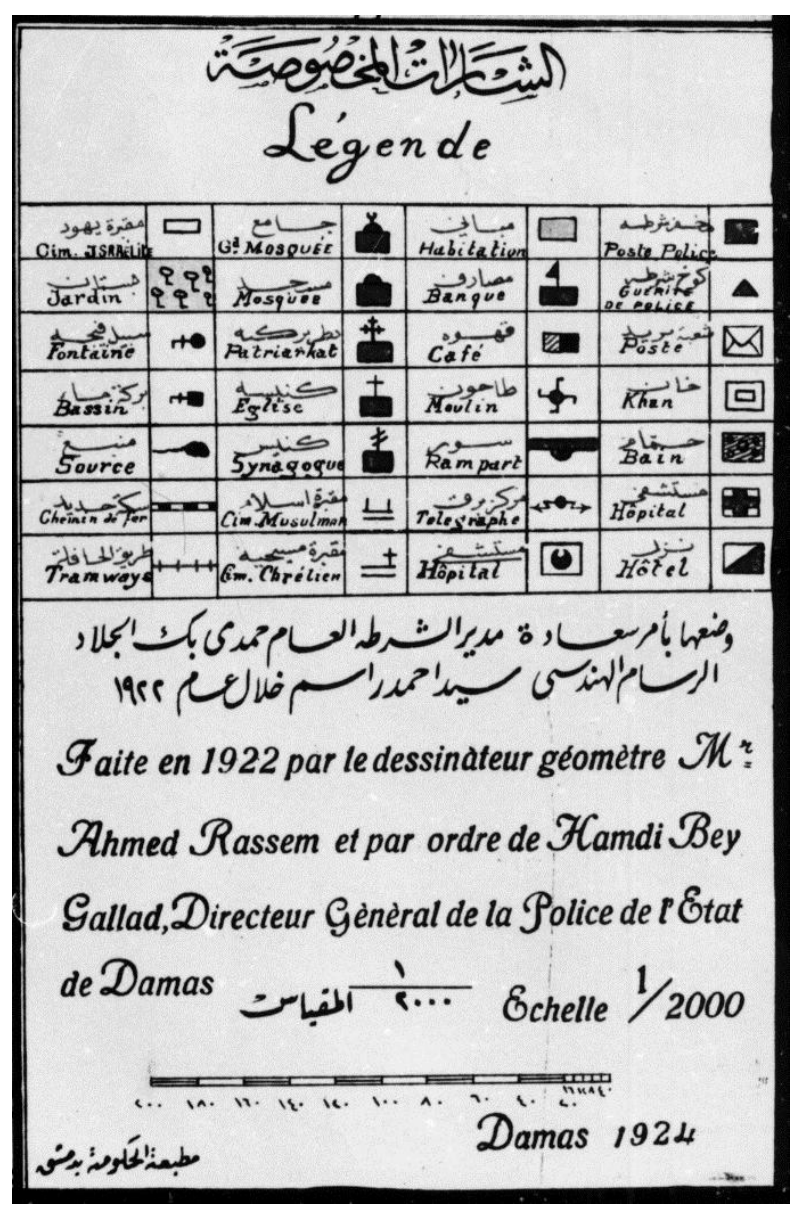

Fig. 15. Légende du plan de Ahmed Rassem, 1922. L'abondance des entrées témoigne de l'attention de l'auteur à l'égard des établissements publics, communautaires ou privés dont la ville est équipée. Extrait de Harila churtâ dimachq (voir note 68), feuille 6, reproduit avec l'autorisation de l'Institut français d'études arabes de Damas.

\section{Ahmed Rassem (1922)}

$\mathrm{Au}$ tout début de la période mandataire, Ahmed Rassem, dessinateur géomètre syrien, est chargé de dresser un plan de Damas par le directeur général de la police de la ville (fig. 14) ${ }^{68}$. Ce document, pour lequel nous disposons seulement d'une photographie en noir et blanc (alors que l'original est en couleurs) conservée à l'institut français d'études arabes de Damas, a été publié en 1924.

L'auteur ne semble pas avoir eu de biographes, en outre, nous ne disposons d'aucune indication quant à la manière dont ce document a été dressé. Sa confrontation avec le plan de Vibert, relevé quelques années plus tard, montre que Rassem disposait du matériel et des connaissances nécessaires à la réalisation d'un plan mesuré et triangulé suivant les règles de l'art ${ }^{69}$, L'échelle qu'il a choisie pour le publier -1:2 000, un centimètre égale vingt mètres - atteste du soin qu'il a apporté à sa réalisation. Ce plan, découpé en six feuilles, est équipé d'une légende très fournie (fig, 15), elle ne compte pas moins de vingthuit entrées qui répertorient à la fois des états de terrain (jardin, cimetière...), des édifices

68. Harita churtâ dimachq [plan de police de Damas], faites (sic) en 1922 par le dessinateur géomètre Mr Ahmed Rassem et par ordre de Hamdi Bey Gallad, Directeur Général de la police de l'Etat de Damas, Damas, Imprimerie du gouvernement, 1924, 1:2 000, six feuilles en couleurs.

69 . Sur quinze mesures comparées, 7 présentent une erreur inférieure à $2 \%$, autant de cas, une erreur comprise entre 2 et $4 \%$ et une erreur de $9,8 \%$ qui témoigne d'une triangulation incomplète et/ou approximative. 


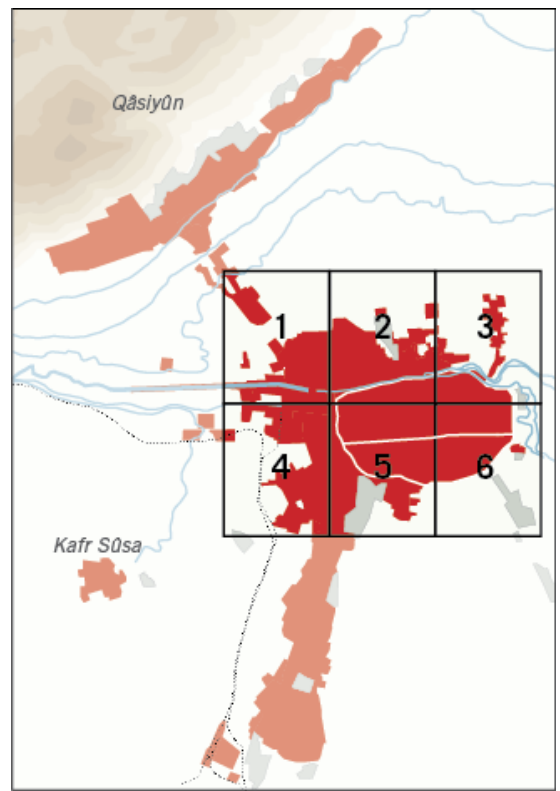

Fig. 16. Périmètre du plan de Ahmed Rassem. Malgré son périmètre restreint, l'échelle de ce plan (1:2 000) en a imposé un découpage en six feuilles. Document établi par l'auteur.

publics et religieux (khân, mosquée, hôtel...) des éléments du réseau hydrographique (source, bassin, fontaine...) ou encore des réseaux de transport (chemin de fer et tramway). La toponymie inscrite directement sur le plan complète cette légende. Elle est bilingue et traite de l'ensemble des noms des voies de circulation, depuis les rues les plus fréquentées jusqu'aux impasses les plus éloignées ${ }^{70}$. Elle mentionne aussi de nombreux noms d'édifices remarquables, de quartiers, de cimetières et ceux des jardins des environs immédiats de l'agglomération. En contrepartie de ces qualités, le territoire couvert, 713 hectares, est assez restreint.

Le plan de Rassem représente l'ensemble de la ville intra-muros, les faubourgs de Châghûr al-barrâniyya, Suwayqa, Qanawât, Sûq Sârûjâ, route d'Alep et une partie de la route de Sâlihiyya, mais il laisse de côté plusieurs centaines d'hectares pourtant compris au sein de l'agglomération avec les quartiers de Mîdân et de Sâlihiyya (fig. 16). Il est remarquable que ce plan s'intéresse assez peu aux quartiers alors en cours d'urbanisation, à l'ouest et au nord de la ville en particulier, pour lesquels une cartographie détaillée aurait facilité la gestion des travaux d'édilité afférent. Mais, au moment où ce plan est publié, une autre entreprise de cartographie, de bien plus grande ampleur, est déjà en cours.

\section{Lucien Vibert (1921, 1924)}

Lucien Vibert est le premier édile damascène chargé de la gestion de l'urbanisation. Il commence ses travaux, suivant une tradition française établie par Haussmann ${ }^{71}$, par le relevé d'un plan de l'ensemble de la ville ${ }^{72}$. Dressé entre 1921 et 1924, c'est le premier plan

70 . La version en caractères latins des désignations a été établie suivant une traduction très approximative pour les toponymes communs (rue correspond à la fois à zuqaq, hara ou tarîq; jardin à jinayna ou bustan) et une translittération tout aussi discutable pour les désignations propres. Ainsi, tarîq sayida zaynab est devenu rue Saidé Zénabe.

71 . Voir à ce propos "Le plan de Paris", dans Mémoires du baron Haussmann - 1853-1870 - Grands travaux de Paris, Paris, Guy Durier, 1979, vol. 1, chap. 1.

72 . Lucien Vibert est ingénieur, il est chef du service technique de la municipalité de Damas à partir de 1922. Franck Fries, "Les plans d'Alep et de Damas, un banc d'essai pour l'urbanisme des frères Danger (1931-1937)", Figures de l'orientalisme en architecture. Revue du monde musulman et de la Méditerranée $\mathrm{n}^{\circ}$ 73-74, 1996, p. 312. 


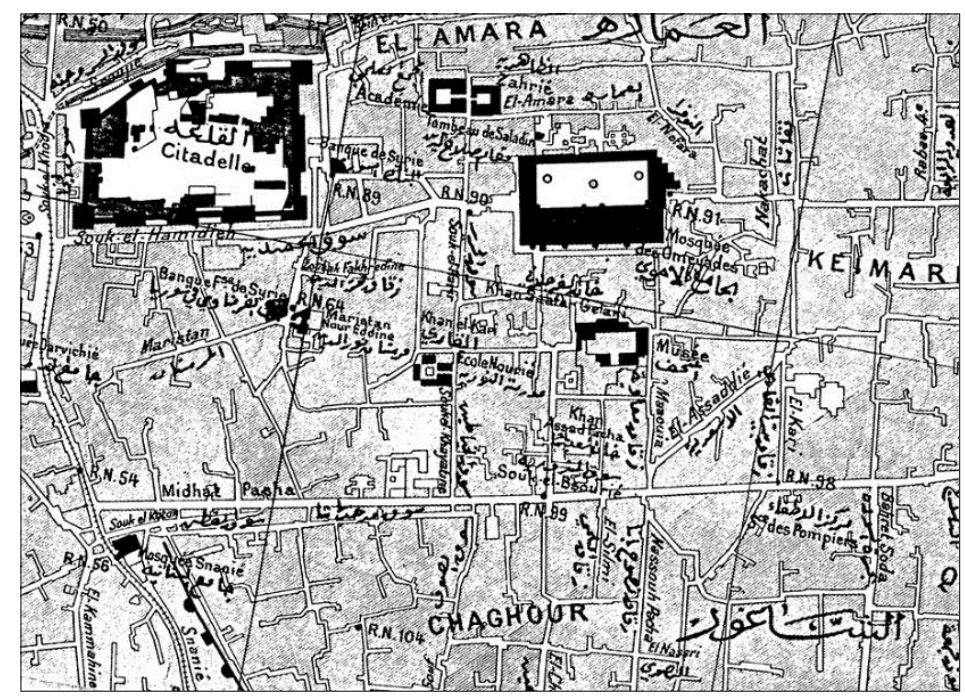

Fig. 17. Plan de Lucien Vibert, 1921-1924, détail. Alors que le plan de Rassem représente la largeur des rues de manière symbolique, Vibert, préoccupé par le bon fonctionnement du réseau, indique avec précision la largeur de chaque section de voie. Extrait de Vibert, Plan de Damas (voir note 32) ; coll. de l'auteur ; même cadrage que les fig. 12 et 14.

de Damas réalisé suivant une base triangulée avec précision. Son élaboration a nécessité trois années de travaux, nous ne disposons pas d'autre information à cet égard, mais sa comparaison avec le plan de 1940 indique de faibles distorsions (fig. 17). Et, malgré son échelle de reproduction : 1:5000 - un centimètre égale cinquante mètres -, il est aussi détaillé que celui de 1922. Il offre plusieurs avantages. Sa date, antérieure aux grands travaux d'urbanisme de la période mandataire, en fait un état des lieux de la ville ottomane ; son échelle de représentation permet de repérer les travaux de régularisation et d'extension du tissu urbain réalisés, dans le cadre de l'administration et de la législation ottomanes, par les gouverneurs successifs de la province, Midhat pacha en particulier au tournant des années $1870-1880^{73}$.

Le plan de Vibert est dressé par et pour les services techniques municipaux. A ce titre, il n'est pas seulement topographique ; sa légende distingue plusieurs modes d'occupation du sol (espaces publics, jardins, cimetières, marais) et elle répartit les constructions entre trois catégories : monuments historiques, monuments publics et constructions diverses. La plupart des établissements publics sont directement nommés sur le plan. Enfin, le dessin du réseau hydrographique est particulièrement soigné puisqu'il figure l'ensemble des canaux, qu'ils soient couverts ou non, information qui n'est pas portée sur les plans plus récents, même les plus précis. Jusqu'au milieu des années trente, ce plan sert de base à l'organisation des travaux d'édilité tels que l'ouverture de nouvelles voies de communication. Le fait qu'il comporte une catégorie "monuments historiques" exprime l'intérêt des nouveaux édiles pour le patrimoine architectural et il n'est pas sans rappeler les plans d'aménagement, d'embellissement et d'extension des villes de France dressés quelques années plus tôt ${ }^{74}$.

73 . Najib E. Saliba, "The Achievements of Midhat Pacha as Governor of the Province of Syria. 1878-1880", International Journal of Middle East Studies $n^{\circ}$ 9:3, 1978, p. 307-323.

74 . Il s'agit de plans imposés aux municipalités dont la population compte plus de 10000 habitants par la loi Cornudet de 1919. Jean-Pierre Gaudin, Les nouvelles politiques urbaines, Paris, Presses universitaires de France, 1993, p. 11-12. 


\section{Une production exogène}

Le plan de Niebuhr est trop sommaire pour constituer une source pour l'histoire de l'urbanisation de Damas. Ainsi, sept documents originaux, assimilables à des plans, rendent compte de l'organisation spatiale de la ville au cours des soixante-dix dernières années de la période ottomane. Malgré ses défauts et ses manques, ce corpus est plus étendu que celui disponible pour les autres villes de la région au cours de la même période. Il témoigne de l'intérêt porté à Damas par les cartographes. Mais, aussi paradoxal que cela puisse paraître, il ne trouve pas son origine dans une volonté de l'autorité publique. Jusqu'à la Première Guerre Mondiale, les plans de Damas sont dressés par des étrangers indépendants du pouvoir ottoman. Ce sont tous des savants qui poursuivent un objectif scientifique et contribuent à l'amélioration de la connaissance de la ville pour un public européen. Durant cette période, l'autorité ottomane se soucie peu de la cartographie de la province de Damas $^{75}$; le premier plan dressé sous la direction de son gouverneur a été publié dans l'annuaire officiel de l'année $1879^{76}$. Il s'agit d'une carte topographique sommaire et aux proportions approximatives, Damas y est représentée par une simple tache très déformée.

Il faut ensuite attendre plus de quarante ans pour que l'administration s'intéresse à la cartographie de la ville avec le plan de 1918. On a vu dans quelles conditions ce document avait été élaboré et on peut se demander s'il ne résulte pas d'une opportunité - la circulation de copies des documents dressés par Wulzinger et Watzinger - plutôt que d'une décision motivée de l'administration. En fait, le premier plan réalisé par un service public et à sa demande est celui de 1922. L'administration mandataire est alors en cours d'installation et c'est dans ce cadre que Ahmed Rassem dresse son plan. Ce document inaugure un nouveau cycle de production. Les suivants sont tous réalisés par les services administratifs mandataires, qu'il s'agisse de la régie du cadastre pour les plans des propriétés ou bien du Service géographique de l'armée pour les cartes plus générales ${ }^{77}$.

75 . Ce qui n'est pas le cas pour la capitale où le sultan demande à un ingénieur allemand, Helmut von Moltke, de dresser un plan dès le milieu des années 1830. Les résultats de son travail sont publiés en ottoman en 1844 Cahit Kayra, Eski lstanbul 'un eski haritalari, lstanbul, lstanbul Büyüksehir belediyesi, 1990, p. 14.

76. Sâlnâma wilâyat Sûrya [annuaire oificiel de la province de Syrie], s.l., Imprimerie-lithographie syrienne, vol. 12, 1297 [1879-1880]), carte placée à la fin du volume.

77 . Cet article constitue la première étape d'un travail consacré à l'urbanisation de Damas à la fin de la période ottomane et conduit avec le soutien de l'institut français d'études arabes de Damas (IFEAD). 